\title{
Advanced Receiver Design for Mitigating Multiple RF Impairments in OFDM Systems: Algorithms and RF Measurements
}

\author{
Adnan Kiayani, Lauri Anttila, Yaning Zou, and Mikko Valkama \\ Department of Communications Engineering, Tampere University of Technology, 33101 Tampere, Finland
}

Correspondence should be addressed to Adnan Kiayani, adnan.kiayani@tut.fi

Received 15 July 2011; Accepted 12 October 2011

Academic Editor: Ming-Der Shieh

Copyright (c) 2012 Adnan Kiayani et al. This is an open access article distributed under the Creative Commons Attribution License, which permits unrestricted use, distribution, and reproduction in any medium, provided the original work is properly cited.

\begin{abstract}
Direct-conversion architecture-based orthogonal frequency division multiplexing (OFDM) systems are troubled by impairments such as in-phase and quadrature-phase (I/Q) imbalance and carrier frequency offset (CFO). These impairments are unavoidable in any practical implementation and severely degrade the obtainable link performance. In this contribution, we study the joint impact of frequency-selective I/Q imbalance at both transmitter and receiver together with channel distortions and CFO error. Two estimation and compensation structures based on different pilot patterns are proposed for coping with such impairments. The first structure is based on preamble pilot pattern while the second one assumes a sparse pilot pattern. The proposed estimation/compensation structures are able to separate the individual impairments, which are then compensated in the reverse order of their appearance at the receiver. We present time-domain estimation and compensation algorithms for receiver I/Q imbalance and CFO and propose low-complexity algorithms for the compensation of channel distortions and transmitter IQ imbalance. The performance of the compensation algorithms is investigated with computer simulations as well as with practical radio frequency $(\mathrm{RF})$ measurements. The performance results indicate that the proposed techniques provide close to the ideal performance both in simulations and measurements.
\end{abstract}

\section{Introduction}

With the ever-increasing demand for high data rates and high quality of services for end users, bandwidth-efficient transmission schemes such as orthogonal frequency division multiplexing (OFDM) are being adopted in emerging wireless communication systems (e.g., WLAN 802.11a/g/n [1], WiMAX IEEE 802.16 [2], DVB-T [3], DVB-H [4], 3GPP LTE [5]). The physical layer implementation of OFDMbased systems with direct-conversion (zero-IF or homodyne) radio architecture represents a promising solution for future wireless systems. The direct-conversion architecture offers a simplified analog front end (FE) as it performs the frequency translation in one step and thus eliminates the need of bulky image rejection filters $[6,7]$. This yields an easy integration of analog and digital components of the FE on a single chip and consequently results in lowercost and less power consumption. From the perspective of practical implementation, a trade-off exists between the high integrability and the performance. The direct-conversion architecture-based transceivers are extremely vulnerable to the nonidealities of analog front-end components. The main impairments that degrade the system performance are inphase quadrature-phase (I/Q) imbalance, DC offset, and carrier frequency offset (CFO) $[6,7]$. The adoption of higher-order modulation alphabets (such as 64-QAM) in OFDM systems suggests that they are increasingly sensitive to any impairments in the underlying analog hardware. Rather than trying to improve the quality of individual analog modules, it is more cost-efficient to tolerate these RF impairments to a certain degree in the analog domain and afterward compensating them in the digital domain.

The frequency up- and downconversion in the directconversion architectures are implemented by I/Q mixing, which suffers from the amplitude and phase mismatch between the I- and Q- branches [8-24]. This problem 
is mainly inflicted by the modulators/demodulators which employ the principle of having equal gain and exact $90^{\circ}$ phase difference between the quadrature branches. However, other analog front-end components such as DACs or ADCs and LPFs also contribute in general to the imbalance effects, resulting in frequency-selective I/Q imbalance [8$10]$. Estimation and compensation schemes dealing with I/Q imbalance have been proposed in several papers including [8-12] and references therein.

CFO is another important RF impairment, particularly associated with OFDM-based communication systems. It is caused by the instability of local oscillator and also due to the mobility of users $[25,26]$. OFDM systems divide the available bandwidth into many orthogonal subcarriers with a small subcarrier spacing. The subcarriers orthogonality is lost in the presence of $\mathrm{CFO}$, leading to intercarrier interference. Because the CFO cannot be perfectly estimated in the presence of I/Q imbalances, most of the recent works (e.g., [18$24,27])$ treat them jointly. The algorithms introduced in [1821 ] analyze the performance degradation and propose compensation scheme coping with CFO and receiver I/Q imbalance, but do not take into account the transmitter I/Q imbalance. Gil et al. [22], Chung and Phoong [23], and Tandur and Moonen [24] developed the joint estimation algorithm of the I/Q imbalance, CFO, and channel, however frequencyindependent I/Q imbalance model is assumed. Finally, in [27], compensation methods for the mitigation of frequencyselective transmitter and receiver I/Q imbalance combined with $\mathrm{CFO}$ and channel distortions are proposed by the authors of this article. But the application of proposed algorithms is restricted to systems with a preamble pilot, and also the computational complexity for $\mathrm{CFO}$ estimation is very large.

In this paper, we consider DSP-based compensation of frequency-selective transmitter and receiver I/Q imbalance together with frequency-selective channel and $\mathrm{CFO}$ in the OFDM system context. The central theme is to first systematically formulate the baseband equivalent of the received signal. Based on this signal model, we propose new low-complexity decoupled estimation and compensation algorithms. Unlike the joint estimation algorithms (e.g., $[22,24]$ ) where the compensator parameters have to be reestimated with channel variations, the decoupled schemes are advantageous in the sense that only the varying parameter has to be reestimated, while the other parameters remain the same. More precisely, at the estimation and compensation stage, we first compensate the generally timeinvariant receiver I/Q imbalance in time domain with blind methods proposed in authors' earlier work [11, 13]. The subsequent estimation and compensation of nonidealities including $\mathrm{CFO}$, channel distortions, and transmitter IQ imbalance is done for pilot symbol-assisted modulation (PSAM) OFDM systems in each transmission. With practical considerations, two pilot patterns are taken into account in this work, namely, the preamble pilot pattern, where one complete OFDM symbol in a transmission frame is assumed known, and the sparse pilot pattern, where pilot symbols are placed over different subcarriers of certain OFDM symbols. We propose two alternative estimation and compensation structures depending on the pilot pattern. The leading principle in CFO estimation and compensation for both pilot patterns is to use an already existing technique of [28] and apply it in time domain. For the preamble pilot-based estimation, we propose zero-forcing (ZF) and maximumlikelihood (ML) estimation methods for joint channel distortions and transmitter I/Q imbalance compensation, whereas an algorithm for successive compensation of channel distortions and transmitter I/Q imbalance is proposed for sparse pilot-based structure. The performance of algorithms is evaluated with extensive computer simulations as well as with laboratory measurement setup.

The novelty of this paper is as follows:

(1) We do not make any specific assumption about the location of pilots for sparse pilot-based estimation and compensation structure. The existing algorithms, for example [29], allocate the pilots to mirror frequency pairs which is generally not valid in practical radio systems like LTE [5].

(2) In this work, instead of proposing comprehensive and brand new algorithms to cope with multiple RF impairments at once, we simplify the complexity of the problem by reforming overall receiver design and decoupling the effects of individual RF impairments. Then incorporating already existing efficient algorithms with new proposed methods, a hybrid time-and-frequency domain compensation architecture with very reasonable complexity and good performance is achieved.

(3) Practical RF measurements are used to verify the applicability of algorithms in real-world receiver design which, to the best of authors' knowledge, has not been addressed so far in the literature. The performance of individual impairments has been evaluated though, for example, $[9,11]$, but RF performance evaluation in the presence of all the considered impairments is still new.

(4) Here we use two widely deployed pilot patterns in the current and upcoming OFDM-based radio systems (e.g., IEEE 802.11n [1], DVB-H/T [3, 4], and LTE [5] systems), namely, the preamble-based and sparsely located pilot structures for parameter estimations. As a result, the proposed algorithms can be directly applied in the corresponding receiver design without any modification.

An attractive feature of receiver I/Q imbalance compensation algorithm is that it is able to track the time-variation of I/Q imbalance and updates the coefficients of compensation filter appropriately.

The paper is organized as follows. I/Q imbalance model and its impact, OFDM signal model under frequencyselective I/Q imbalances, channel distortions, and CFO are described in Section 2. Section 3 presents the estimation and compensation techniques for the mitigation of impairments. Computer simulation results are shown in Section 4. In Section 5, the measurement setup and obtained results are presented. Finally, the conclusions are drawn in Section 6. 
Preliminaries. The notations used in this paper are as follows. Scalar parameters are represented by lower case letters $a$ and frequency domain quantities with upper case letters $A$. We denote the time domain vectors/matrices by lower case bold face with over-bar $\overline{\mathbf{a}} / \overline{\mathbf{A}}$ and frequency domain vectors/matrices by bold face letters $\mathbf{a} / \mathbf{A}$. Superscript $(\cdot)^{T},(\cdot)^{*}$, and $(\cdot)^{-1}$ denote the transpose, conjugate, and inverse of a vector, a scalar, or a matrix, respectively. The $(i, j)$ th quantity of a matrix is defined by $A_{i j}$ and $(i, i)$ th quantity by $A_{i}$. The convolution operation is indicated by $\star$. If $\mathbf{a}=\left[a_{1}, a_{2}, \ldots, a_{N / 2}, \ldots, a_{N}\right]^{T}$ is an OFDM symbol with $N$ subcarriers then $\mathbf{a}^{\#}=\left[a_{1}^{*}, a_{N}^{*}, \ldots, a_{N / 2}^{*}, \ldots, a_{2}^{*}\right]^{T}$ is the conjugate of mirror subcarriers vector. The discrete Fourier transform operation is denoted by a matrix $\mathbf{F}$ of size $N \times N$ defined as $[\mathbf{F}]_{m n}=e^{-j(2 \pi m n / N)}, m=0,1, \ldots, N-1 ; n=$ $0,1, \ldots, N-1$.

\section{Signal and System Model}

2.1. Frequency-Selective I/Q Imbalance Model. The directconversion transmitter architecture is based on the principle of directly I/Q upconverting the baseband signal to the RF frequency. The upconversion is performed in the analog domain by a quadrature mixer, which theoretically provides infinite image signal attenuation. This eliminates the need for image rejection filter, relaxing the overall requirement for RF filtering. A perfectly balanced modulator corresponds to equal gain and $90^{\circ}$ phase difference between the quadrature branches. However, in practice, this requirement is not fully satisfied. In addition to that, other modem components such as DACs and LPFs in the I- and Q- branch are not perfectly matched $[7,8]$. These effects are called I/Q imbalance, and it results in the limited suppression of the image signal $[8,9]$. In the wideband system context, the reconstruction filters in the modulator exhibit frequency-dependent response, which causes the I/Q imbalance to vary as a function of frequency. Consequently, we can characterize the I/Q imbalance with transmitter gain imbalance and phase imbalance parameter $g_{T}$ and $\phi_{T}$, respectively $[8,27]$. In addition, the relative nonideal filter transfer function between in the I- and Qbranches is modeled with the filter $h_{T}(t)$. A conceptual block diagram is illustrated in Figure 1. To see the impact of I/Q imbalance on the transmitted signal, we denote the baseband equivalent ideal transmit signal as $z(t)=z_{I}(t)+j z_{Q}(t)$. The complex envelope of the transmitted RF signal with $\mathrm{I} / \mathrm{Q}$ imbalance effects is [8]

$$
s(t)=g_{1, T}(t) \star z(t)+g_{2, T}(t) \star z^{*}(t),
$$

where the complex filters $g_{1, T}(t)$ and $g_{2, T}(t)$ correspond to imbalance filters and are defined as

$$
\begin{aligned}
& g_{1, T}(t)=\frac{\delta(t)+g_{T} \exp \left(j \phi_{T}\right) h_{T}(t)}{2}, \\
& g_{2, T}(t)=\frac{\delta(t)-g_{T} \exp \left(j \phi_{T}\right) h_{T}(t)}{2} .
\end{aligned}
$$

The baseband equivalent signal in frequency domain is

$$
S(f)=G_{1, T}(f) Z(f)+G_{2, T}(f) Z^{*}(-f) .
$$

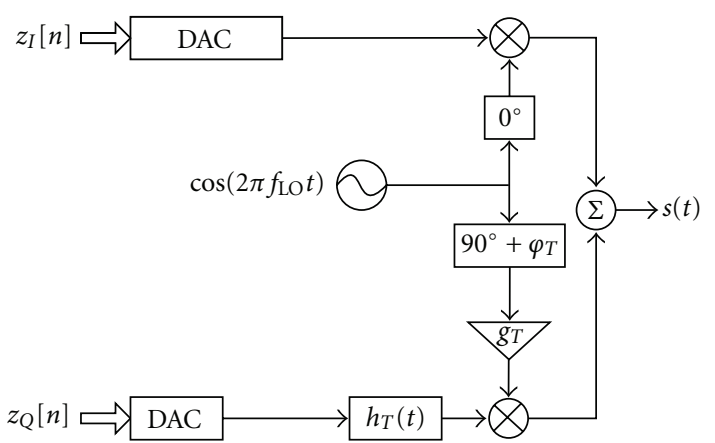

FIGURE 1: Block diagram of Frequency-selective transmitter I/Q imbalance model.

The above equation indicates that the imbalanced baseband signal is weighted sum of the desired signal $Z(f)$ and the undesired image signal $Z^{*}(-f)$. The undesired image signal term is produced by the I/Q imbalance and results in the mirror frequency interference.

On the receiver side, the RF signal is downconverted to baseband using a quadrature demodulator. The downconverted baseband equivalent of the received signal $r(t)$ is of the form [11]

$$
x(t)=g_{1, R}(t) \star r(t)+g_{2, R}(t) \star r^{*}(t)
$$

with the receiver I/Q imbalance filters $g_{1, R}(t)$ and $g_{2, R}(t)$ given as

$$
\begin{aligned}
g_{1, R}(t) & =\frac{\delta(t)+g_{R} \exp \left(-j \phi_{R}\right) h_{R}(t)}{2}, \\
g_{2, R}(t) & =\frac{\delta(t)-g_{R} \exp \left(j \phi_{R}\right) h_{R}(t)}{2} .
\end{aligned}
$$

The Fourier transform of (4) is

$$
X(f)=G_{1, R}(f) R(f)+G_{2, R}(f) R^{*}(-f) .
$$

Again the mirror-frequency interference due to I/Q imbalance is evident from (6).

The image rejection ratio (IRR) quantifies the image signal suppression and is defined as the ratio of desired signal power to image signal power, expressed in $\mathrm{dB}$ as

$$
\operatorname{IRR}_{T ; R}(f) \triangleq 10 \log _{10}\left(\frac{\left|G_{1, T ; R}(f)\right|^{2}}{\left|G_{2, T ; R}(f)\right|^{2}}\right)[d B] .
$$

With careful analog design, IRR's in the order of $25-40 \mathrm{~dB}$ are stated feasible [6-8].

2.2. OFDM Signal Model with RF Impairments. We consider an OFDM system with $N$ subcarriers. At the transmitter, OFDM symbols are generated by computing the $N$-point inverse discrete Fourier transform (IDFT) of the data symbols $\mathbf{u}$. A cyclic prefix $(\mathrm{CP})$ of length $L_{\mathrm{CP}}$ is appended ahead of each OFDM symbol which is removed at the receiver after DFT. 


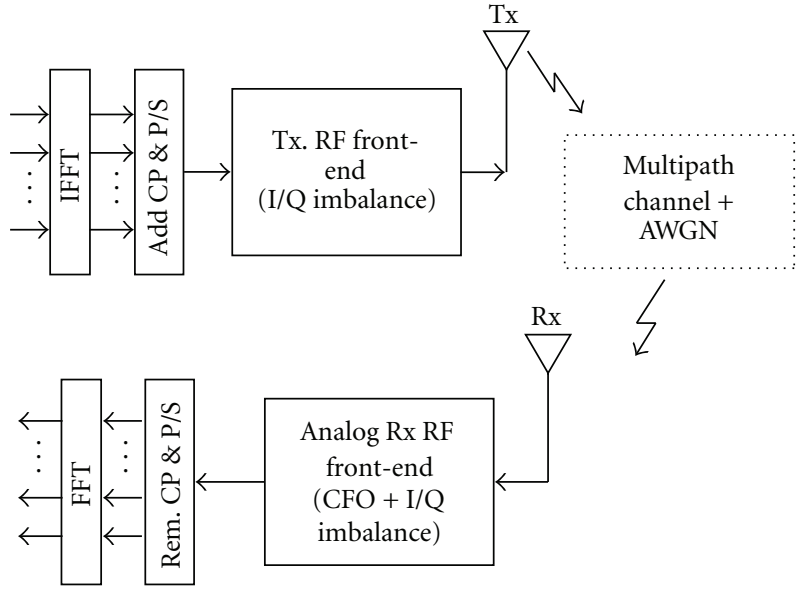

FIgURE 2: OFDM system model with I/Q imbalance and CFO.

Referring to Figure 2, consider the transmission of $i$ th symbol of size $N \times 1$, the frequency domain transmitted signal corresponding to $i$ th OFDM symbol is then given by

$$
\mathbf{s}^{i}=\mathbf{G}_{1, T} \mathbf{u}^{i}+\mathbf{G}_{2, T} \mathbf{u}^{i \#}
$$

where $\mathbf{G}_{1, T}$ and $\mathbf{G}_{2, T}$ are $N \times N$ frequency domain diagonal matrices whose diagonal elements possess frequency responses of transmitter I/Q imbalance filters $\left(g_{1, T} ; g_{2, T}\right.$ in (2)). We consider the transmission over a block fading channel whose impulse response is denoted by $\overline{\mathbf{h}}=[h(0), h(1), \ldots, h(L-1)]^{T}$ with channel length $L$. The received frequency domain signal can then be expressed as

$$
\mathbf{r}^{i}=\mathbf{H G}_{1, T} \mathbf{u}^{i}+\mathbf{H G}_{2, T} \mathbf{u}^{i \#}+\mathbf{n},
$$

where $\mathbf{H}$ is $N \times N$ frequency domain matrix that contains the channel frequency response on its main diagonal and $\mathbf{n}$ is additive white Gaussian noise (AWGN).

Assume now that the local oscillator of transmitter and receiver are not synchronized and a CFO $\Delta f$ is present. If we denote the time domain received signal vector as $\overrightarrow{\mathbf{r}}^{i}$, then the signal in the presence of CFO can be modeled as $\overline{\mathbf{r}}^{i^{\prime}}=\bar{\Omega} \overline{\mathbf{r}}^{i}$, where $\bar{\Omega}^{i}=\operatorname{diag}\left\{\overline{\mathbf{c}^{i}}\right\}$ is the CFO matrix with main diagonal entries

$$
\overline{\mathbf{c}}^{i}=e^{j 2 \pi \Delta f T_{s}\left\{(i-1) N+i L_{\mathrm{CP}}\right\}} \times\left[1, e^{j 2 \pi \Delta f T_{s}}, \ldots, e^{j 2 \pi \Delta f T_{s}(N-1)}\right]^{T} .
$$

Here $\Delta f$ is the frequency offset in Hertz and $T_{s}$ is the sampling rate. The frequency domain $N \times N$ CFO matrix $\Omega^{i}=\mathbf{F} \bar{\Omega}^{i}$ is circulant in nature and has $c(0)$ on its main diagonal and $c^{i}(1)$ and $c^{i}(N-1)$ on the first sub-and superdiagonal [30]. Including the CFO effect to (9), we obtain

$$
\mathbf{r}^{i}=\Omega^{i} \mathbf{H G}_{1, T} \mathbf{u}^{i}+\Omega^{i} \mathbf{H G}_{2, T} \mathbf{u}^{i \#}+\mathbf{n} .
$$

Finally taking into account the receiver I/Q imbalance, the signal at the downconversion stage appears as

$$
\mathbf{x}^{i}=\mathbf{G}_{1, R} \mathbf{r}^{i}+\mathbf{G}_{2, R} \mathbf{r}^{i \#},
$$

where again $\mathbf{G}_{1, R}$ and $\mathbf{G}_{2, R}$ are $N \times N$ frequency domain diagonal matrices whose diagonal elements are DFT of receiver I/Q imbalance filters $\left(g_{1, R} ; g_{2, R}\right.$ in (5)). After some simple mathematical manipulations, the complex baseband equivalent of the received signal in terms of transmitter/receiver I/Q imbalance, channel, and CFO is given by

$$
\begin{aligned}
\mathbf{x}^{i}= & \left(\mathbf{G}_{1, R} \Omega^{i} \mathbf{H G}_{1, T}+\mathbf{G}_{2, R} \Omega^{i \#} \mathbf{H}^{\#} \mathbf{G}_{2, T}^{\#}\right) \mathbf{u}^{i} \\
& +\left(\mathbf{G}_{1, R} \Omega^{i} \mathbf{H G}_{2, T}+\mathbf{G}_{2, R} \Omega^{i \#} \mathbf{H}^{\#} \mathbf{G}_{1, T}^{\#}\right) \mathbf{u}^{i \#}+\mathbf{v},
\end{aligned}
$$

where $\Omega^{i \#}, \mathbf{H}^{\#}, \mathbf{G}_{1, T}^{\#}, \mathbf{G}_{2, T}^{\#}$ are the mirrored matrix of $\Omega^{i}, \mathbf{H}, \mathbf{G}_{1, T}, \mathbf{G}_{2, T}$, respectively. The noise term now becomes $\mathbf{v}=\mathbf{G}_{1, R} \mathbf{n}+\mathbf{G}_{2, R} \mathbf{n}^{\#}$, whose elements are still complex circular Gaussian, but with correlated mirror subcarriers.In the ideal case when there is perfect transmitter/receiver modulator/demodulator matching as well as perfect synchronization between the transmitter/receiver local oscillator, the model in (13) reduces to $\mathbf{x}^{i}=\mathbf{H u}^{i}+\mathbf{n}$ which can be equalized with one complex multiplication for each subcarrier in each OFDM symbol. As evident from (13), both I/Q imbalances and CFO introduce ICI and severely degrade the system performance, thus digital compensation is needed.

In this paper, we assume that the length of the cascade of impulse responses of the transmitter I/Q imbalance filters $\left(g_{1, T}(t)\right.$ and $g_{2, T}(t)$ in $\left.(1)\right)$, the multipath radio channel, and the receiver I/Q imbalance filters $\left(g_{1, R}(t)\right.$ and $g_{2, R}(t)$ in $\left.(4)\right)$ does not exceed the length of CP. It is reasonable to make such an assumption as the frequency-selective transceiver I/Q imbalance is rather moderate and can be estimated with sufficient accuracy with only a few taps $[8,11,27]$.

\section{Estimation and Compensation of Nonidealities}

The estimation and compensation of radio frequency impairments in OFDM systems can be performed blindly and/or with the aid of training (a.k.a. pilot) symbols. For forming such pilot-symbol-assisted OFDM systems, it involves inserting the known symbols in the stream of data symbols. With practical system design, two types of pilot patterns are widely used-the preamble pilot pattern that amends an entirely known OFDM symbol at the beginning of the frame and the sparse pilot pattern where pilot symbols are sparsely inserted at some subcarriers of specific OFDM symbols. Figure 3 illustrate these two ways of inserting pilots among the data symbols.

In the following, we discuss receiver-based estimation and compensation algorithms for mitigating all the considered RF impairments. With developed signal model and given pilot patterns, we are able to isolate them individually and then process them for estimation and compensation in the reverse order of their appearance in the transmitter and receiver front end. We first discuss the receiver I/Q imbalance estimation and compensation, which is performed in time domain and is independent of the pilot pattern. Then, two low-complexity and novel structures are proposed for CFO, channel, and transmitter I/Q imbalance compensation with both preamble and sparse pilot structures. The CFO 


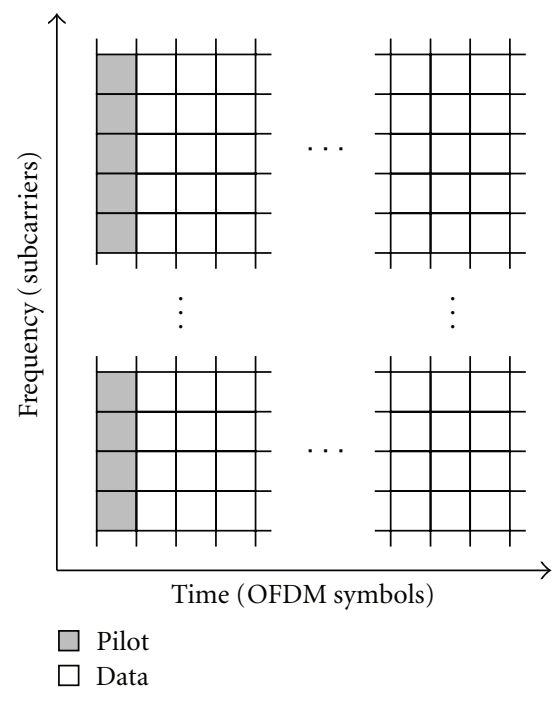

(a)

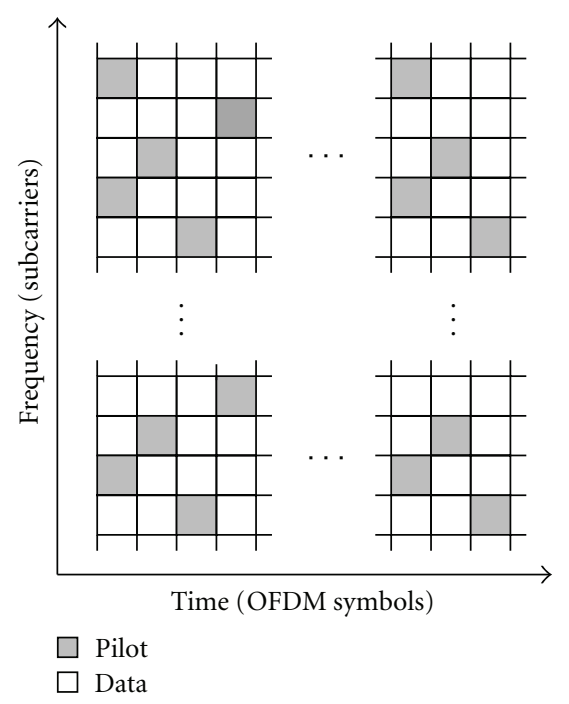

(b)

Figure 3: Pilot structure (a) preamble (b) scattered.

estimation and compensation is also carried out in time domain, while the channel and transmitter IQ imbalance are compensated in frequency domain.

3.1. Blind Receiver I/Q Imbalance Compensation. For receiver I/Q imbalance estimation and compensation, we propose to utilize the statistical signal processing-based blind I/Q imbalance compensation algorithms, developed in authors' earlier work $[11,13,14]$. In general, most complex communication waveforms based on $M$-PSK and $M$-QAM (with $M>2$ ) are proper or circular [31]. The OFDM signal constructed from these alphabets is thus also circular. I/Q imbalance destroys this property and makes the signal noncircular. The strategy used in [11] is to recover the circularity using second-order statistics-based approach, under the assumption that the received signal (without receiver I/Q imbalance) is circular. The circularity of received signal is inherently lost in the presence of transmitter I/Q imbalance, potentially hindering the applicability of the I/Q imbalance compensation algorithms of [11]. However, it is shown in [13] that either a CFO or a fading channel will restore the circularity of the received signal (without receiver I/Q imbalance), thus making it possible to utilize the circularity-based algorithms of [11]. These blind algorithms are also beneficial from the point of view that they do not affect the subsequent signal processing of $\mathrm{CFO}$, channel, and transmitter I/Q imbalance estimation and compensation.

For notational simplicity, we drop the noise term in (13) and continue the analysis. Notice that even though the actual compensation processing is done partly in the time domain (for receiver I/Q imbalance and CFO), the following analysis is done completely in the frequency domain. From (12), we recognize that the signal $\mathbf{x}^{i}$ is the linear combination of the received signal $\mathbf{r}^{i}$ and its mirror conjugate $\mathbf{r}^{i \#}$. For such a system model, the natural compensator is of the form

$$
\mathbf{y}^{i}=\mathbf{x}^{i}+\mathbf{W}_{R} \mathbf{x}^{i \#} \text {. }
$$

Substituting the observed signal $\mathbf{x}^{i}$ of (12) into the compensator in (14), the output can be written as

$$
\mathbf{y}^{i}=\left(\mathbf{G}_{1, R}+\mathbf{W}_{R} \mathbf{G}_{2, R}^{\#}\right) \mathbf{r}^{i}+\left(\mathbf{G}_{2, R}+\mathbf{W}_{R} \mathbf{G}_{1, R}^{\#}\right) \mathbf{r}^{i \#} .
$$

From (15), it follows that the optimum compensation filter $\mathbf{W}_{R}$ canceling the mirror-frequency interference is, in frequency domain $N \times N$ diagonal matrix, of the form $\mathbf{W}_{\mathrm{OPT}}=-\mathbf{G}_{2, R}\left(\mathbf{G}_{1, R}^{\#}\right)^{-1}$ and is derived in [11].

Applying the optimum compensation filter to (15) cancels the mirror conjugate term $\mathbf{r}^{i *}$ and results in the output signal as

$$
\begin{aligned}
\mathbf{y}^{i}= & \left(\mathbf{G}_{1, R} \Omega^{i} \mathbf{H G}_{1, T}-\mathbf{G}_{2, R} \mathbf{G}_{2, R}^{\#}\left(\mathbf{G}_{1, R}^{\#}\right)^{-1} \Omega^{i} \mathbf{H G}_{1, T}\right) \mathbf{u}^{i} \\
& +\left(\mathbf{G}_{1, R} \Omega^{i} \mathbf{H G}_{2, T}-\mathbf{G}_{2, R} \mathbf{G}_{2, R}^{\#}\left(\mathbf{G}_{1, R}^{\#}\right)^{-1} \Omega^{i} \mathbf{H G}_{1, T}\right) \mathbf{u}^{i \#} .
\end{aligned}
$$

It is well known that $\left|\mathbf{G}_{1 ; T, R}\right|>\left|\mathbf{G}_{2 ; T, R}\right|$ and $\left|\mathbf{G}_{1 ; T, R}\right| \approx 1$ for any practical imbalance values; thus the contributions of the second terms inside the parentheses are relatively very small compared to the first term and can be omitted, leading to the following form:

$$
\mathbf{y}^{i} \approx \mathbf{G}_{1, R} \Omega^{i} \mathbf{H}\left(\mathbf{G}_{1, T} \mathbf{u}^{i}+\mathbf{G}_{2, T} \mathbf{u}^{i \#}\right) .
$$

Comparing (16) with (13) suggests that, given $\mathbf{W}_{\mathrm{OPT}}$, the compensator is able to fully suppress the image signal term generated by a frequency-selective receiver I/Q imbalance in the presence of CFO, channel distortions, and transmitter I/Q imbalance. The estimation of $\mathbf{W}_{\mathrm{OPT}}$ is described in $[11,13]$ and is not reproduced here. Next, we discuss the pilot-based estimation and compensation schemes for CFO, channel distortions, and transmitter I/Q imbalance.

3.2. Estimation and Compensation with Preamble Pilot Structure. The compensation structure proposed in this section 


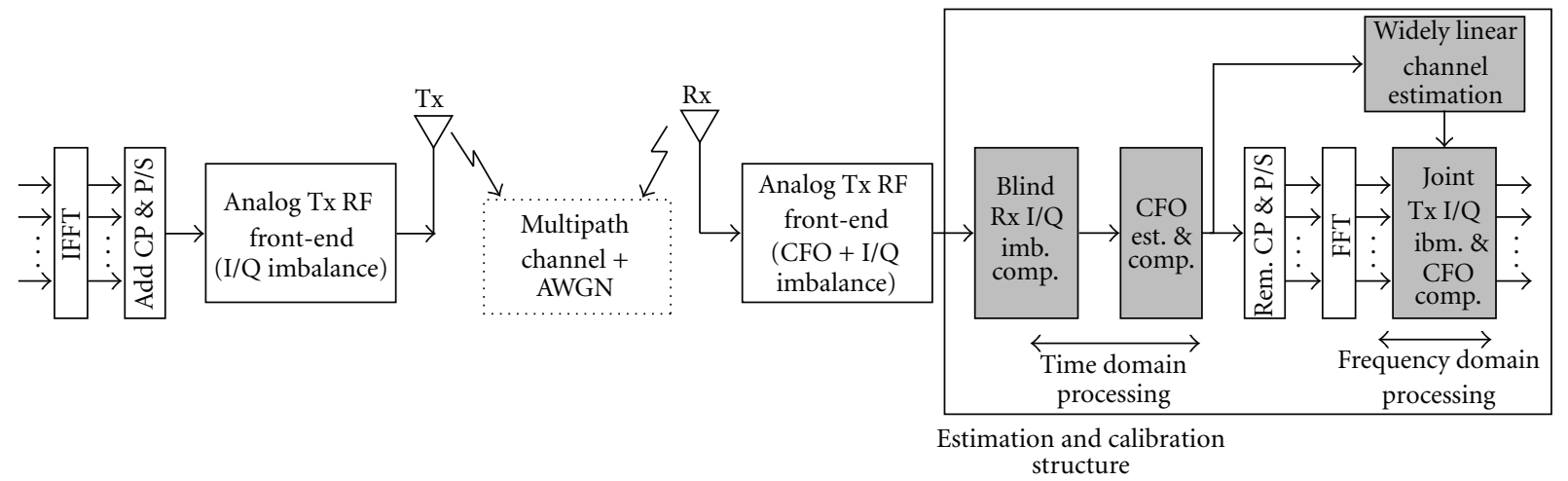

FIGURE 4: OFDM system model with preamble pilot compensator structure.

utilizes one complete pilot OFDM symbol embedded in the OFDM frame. The preamble pilot symbol is transmitted at the beginning of the transmission to estimate the RF impairments. This kind of pilot pattern assumes that the channel remains static over several OFDM symbols and has the benefit of efficiently using the available bandwidth. OFDMbased systems such as IEEE $802.11 \mathrm{n}$ and IEEE $802.16 \mathrm{~d}$ have a preamble at the beginning of the frame [25]. Figure 4 shows the system model with RF impairments estimation and compensation block in place. In the following, we discuss the algorithms for the estimation and compensation of RF impairments.

3.2.1. CFO Estimation and Compensation. There exists an abundant literature on CFO estimation (see, e.g, [18-25] and references therein), but most of these algorithms assume a specific pilot structure which restrict their use in our problem. The subspace-based CFO estimation approach proposed in [26] utilizes only one OFDM symbol, and can be directly used here at the expense of increased computational complexity. We rather suggest to use the CFO estimation algorithm of [28] as discussed in [25]. More specifically, the actual CFO estimation is performed in two stages: first the fractional part of CFO is estimated and corrected in time domain by using the $\mathrm{CP}$ correlation. The integer part is then estimated in frequency domain in the second stage. Even though the presence of transmitter I/Q imbalance is not taken into account in this method, we show with simulations that the algorithm is still able to achieve reasonable good CFO estimation performance.

Assuming perfect $\mathrm{CFO}$ estimate is obtained, we perform CFO compensation in time domain by multiplying the time domain equivalent of (17) with the complex conjugate of $\bar{\Omega}^{i}$, which, in time domain, can be written as

$$
\begin{aligned}
\widetilde{\mathbf{y}}^{i} & =\mathbf{F}\left[\bar{\Omega}^{i *} \overline{\mathbf{y}}^{i}\right] \\
& =\widetilde{\mathbf{G}}_{1, R} \mathbf{H}\left(\mathbf{G}_{1, T} \mathbf{u}^{i}+\mathbf{G}_{2, T} \mathbf{u}^{i \#}\right),
\end{aligned}
$$

where $\widetilde{\mathbf{G}}_{1, R} \triangleq \Omega^{i \#} \mathbf{G}_{1, R}$.

3.2.2. Joint Transmitter I/Q Imbalance and Channel Equalization. The joint transmitter I/Q imbalance and channel equalization are carried out in frequency domain. To simplify the analysis further, let us define the direct and image signal filters as $\mathbf{G}_{D} \triangleq \widetilde{\mathbf{G}}_{1, R} \mathbf{H G}_{1, T}$ and $\mathbf{G}_{M} \triangleq \widetilde{\mathbf{G}}_{1, R} \mathbf{H G}_{2, T}$, which transforms (18) into

$$
\tilde{\mathbf{y}}^{i}=\mathbf{G}_{D} \mathbf{u}^{i}+\mathbf{G}_{M} \mathbf{u}^{i *}
$$

We now switch to the subcarrier model and write the CFO compensated symbol for the $k$ th subcarrier and the complex conjugate of its mirror subcarrier. In the subsequent derivations, the subcarrier index $-k$ refers to the physically opposite mirror subcarrier

$$
\left[\begin{array}{c}
\tilde{Y}_{k}^{i} \\
\tilde{Y}_{-k}^{i *}
\end{array}\right]=\underbrace{\left[\begin{array}{cc}
G_{D, k} & G_{M, k} \\
G_{M,-k}^{*} & G_{D,-k}^{*}
\end{array}\right]}_{G_{\text {total }, k}}\left[\begin{array}{c}
U_{k}^{i} \\
U_{-k}^{i *}
\end{array}\right] .
$$

The matrix $G_{\text {total }, k}$ represents the joint transmitter I/Q imbalance and channel response. Assuming the coefficients of matrix $G_{\text {total }, k}$ are known, we are able to directly apply the $\mathrm{ZF}$ or the ML detection principle on the mirror subcarrier pair to estimate the transmitted symbol.

With ZF equalization scheme, the estimate of original transmit symbols is obtained by solving (20) for each subcarrier $k$ and its mirror subcarrier $-k$ as

$$
\left[\begin{array}{c}
\hat{U}_{k}^{i} \\
\hat{U}_{-k}^{i *}
\end{array}\right]=\hat{G}_{\text {total }, k}^{-1}\left[\begin{array}{c}
\tilde{Y}_{k}^{i} \\
\tilde{Y}_{-k}^{i *}
\end{array}\right] .
$$

It is well known that ZF equalization suffers from the noise enhancement problem, yet it gives good calibration performance as will be shown in Section 4.

Alternatively, the transmitted symbols can be estimated using ML detection principle which is based on the principle of minimizing the cost function

$$
\left[\begin{array}{c}
\hat{U}_{k}^{i} \\
\hat{U}_{-k}^{i *}
\end{array}\right]=\arg \min _{\breve{U}_{k}^{i}, \breve{U}_{-k}^{i *}}\left\|\hat{G}_{\text {total }, k}\left[\begin{array}{c}
\breve{U}_{k}^{i} \\
\breve{U}_{-k}^{i *}
\end{array}\right]-\left[\begin{array}{c}
\tilde{Y}_{k}^{i} \\
\widetilde{Y}_{-k}^{i *}
\end{array}\right]\right\|^{2},
$$

where $\breve{U}_{k}^{i}$ in (22) denotes the trial value of OFDM symbol $U_{k}^{i}$. The ML equalizer is able to exploit the frequency diversity induced by transmitter I/Q imbalance and gives better performance than $\mathrm{ZF}$, but the computational complexity associated with ML is very large which increases with higherorder constellations and large number of subcarriers. 
3.2.3. LS Identification of $G_{\text {total } K \text {. We consider the LS estima- }}$ tion of the joint transmitter I/Q imbalance and channel filters $\mathbf{G}_{D}$ and $\mathbf{G}_{M}$. The filters are estimated in time domain similar to [32], where a time-domain ML channel estimator was proposed for OFDM. This approach can utilize the frequency domain correlation of the channel implicitly without making any statistical assumptions about the channel, through limiting the length of the estimated channel response estimates as $N_{g} \ll N$.

We switch to matrix-vector algebra and write the time-domain received symbol corresponding to preamble pilot as $\overline{\widetilde{\mathbf{y}}}^{p}(n) \triangleq\left[\tilde{y}^{p}(n), \tilde{y}^{p}(n-1), \ldots, \tilde{y}^{p}(n-N+1)\right]^{T}$ and the observable imbalance filters as $\overline{\mathbf{g}}_{D ; M}=$ $\left[\widehat{g}_{D ; M, 1}, \hat{g}_{D ; M, 2}, \ldots, \hat{g}_{D ; M, N_{g}}\right]^{T}$, where $N$ is the length of pilot data and $N_{g}<L_{\mathrm{CP}}$ is the length of estimated filters. The received symbol vector during the pilot symbol is then

$$
\begin{aligned}
\overline{\tilde{\mathbf{y}}}^{p}(n) & =\overline{\mathbf{U}}^{p}(n) \overline{\mathbf{g}}_{D}+\overline{\mathbf{U}}^{p *}(n) \overline{\mathbf{g}}_{M} \\
& =\left[\overline{\mathbf{U}}^{p}(n) \overline{\mathbf{U}}^{p *}(n)\right]\left[\begin{array}{l}
\overline{\mathbf{g}}_{D} \\
\overline{\mathbf{g}}_{M}
\end{array}\right] \\
& =\overline{\mathbf{U}}_{b}^{p}(n)\left[\begin{array}{c}
\overline{\mathbf{g}}_{D} \\
\overline{\mathbf{g}}_{M}
\end{array}\right],
\end{aligned}
$$

where $\overline{\mathbf{U}}^{p}(n)$ is the time-domain circular-convolution matrix formed from IDFT of pilot symbol $\mathbf{u}_{p}$. The LS estimates of imbalance filters can be computed as [33]

$$
\left[\begin{array}{c}
\overline{\mathbf{g}}_{D} \\
\hat{\mathbf{g}}_{M}
\end{array}\right]=\overline{\mathbf{U}}_{b}^{p \dagger}(n) \tilde{\overline{\mathbf{y}}}^{p}(n) .
$$

Here $\overline{\mathbf{U}}_{b}^{p \dagger}(n)$ represents the pseudoinverse of $\overline{\mathbf{U}}_{b}^{p}(n)$ and is given by $\overline{\mathbf{U}}_{b}^{p \dagger}(n)=\left(\overline{\mathbf{U}}_{b}^{p H}(n) \overline{\mathbf{U}}_{b}^{p}(n)\right)^{-1} \overline{\mathbf{U}}_{b}^{p H}(n)$ [33]. Frequency domain expressions of the estimated filters $\mathbf{G}_{D}$ and $\mathbf{G}_{M}$, which are needed for equalization/detection in (20)-(22), are then obtained through DFT.

\subsection{Estimation and Compensation with Sparse Pilot Structure.} OFDM systems such as LTE and DVB-T/H do not include a preamble pilot in their frame, rather the pilot tones are inserted sparsely in the OFDM symbols. DVB-T/H standard defines both continual and scattered pilots, on the other hand, LTE includes pilot subcarriers only on specified OFDM symbols. Figure 5 shows the reference pilot structure for LTE system with pilot symbols at every sixth subcarrier during the first and fifth OFDM symbol of each slot.

One big challenge with sparse located pilot structure is that the pilots are most likely not allocated to mirrorfrequency pairs which is required by most of the pilot-based algorithms developed in literature, for example, [29]. The compensation algorithms proposed in this section consist of estimating the impairments at the pilot frequencies and then interpolating the estimates over all the subcarriers. Therefore, no pilot structure modification is needed, and they can be directly applied for the development of, for example, LTE receiver. The estimation and compensation structure is illustrated in Figure 6. Again, notice that the receiver I/Q imbalance has already been compensated with the blind method discussed in Section 3.1.

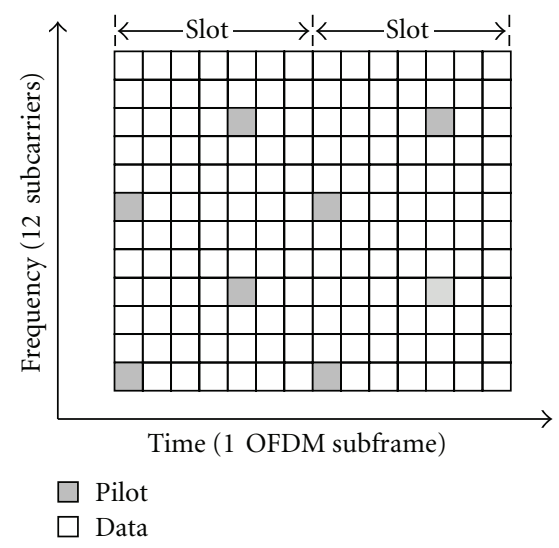

FIGURE 5: LTE reference pilot structure.

3.3.1. CFO Estimation and Compensation. For the CFO estimation, we again apply the two-step time-domain approach of [25] as discussed in the previous subsection, resulting in the time domain CFO compensated signal similar to (18) as

$$
\begin{aligned}
\tilde{\mathbf{y}}^{i} & =\widetilde{\mathbf{G}}_{1, R} \mathbf{H G}_{1, T} \mathbf{u}^{i}+\widetilde{\mathbf{G}}_{1, R} \mathbf{H G}_{2, T} \mathbf{u}^{i \#} \\
& =\mathbf{G}_{D} \mathbf{u}^{i}+\mathbf{G}_{M} \mathbf{u}^{i \#} .
\end{aligned}
$$

\subsubsection{Decoupled Transmitter I/Q Imbalance and Channel} Equalization. With the sparse pilot structure, we propose to compensate the channel and transmitter I/Q imbalance successively. Algorithm 1 summarizes the algorithm for the estimation of transmitted symbols.

Algorithm 1 (It is for estimation of channel distortions and transmitter I/Q imbalance).

(1) Estimate widely-linear filter $G_{D, k}$ (see Section 3.3.3).

(2) Switch to subcarrier model and write (25) in frequency domain for subcarrier $k$ as

$$
\tilde{Y}_{k}^{i}=G_{D, k} U_{k}^{i}+G_{M, k} U_{-k}^{i *} .
$$

(3) Divide (26) by $G_{D, k}$ to obtain the equalized subcarriers as

$$
\begin{aligned}
\widetilde{S}_{k}^{i} & =\frac{1}{G_{D, k}} \tilde{Y}_{k}^{i}=U_{k}^{i}+\frac{G_{M, k}}{G_{D, k}} U_{-k}^{i *} \\
& =U_{k}+\frac{G_{2, T, k}}{G_{1, T, k}} U_{-k}^{i *} .
\end{aligned}
$$

Clearly, the channel effect is removed and the only impairment present in the signal now is the transmitter I/Q imbalance.

(4) Hard-decision-based detection of the mirror subcarriers of equalized symbol, given by

$$
\breve{S}_{-k}^{i}=D\left(\widetilde{S}_{-k}^{i}\right),
$$

where $D$ denotes the decision.

(5) At the pilot subcarriers, we now have

$$
\widetilde{S}_{k}^{p}=U_{k}^{p}-W_{k}^{p} \breve{S}_{-k}^{p} .
$$




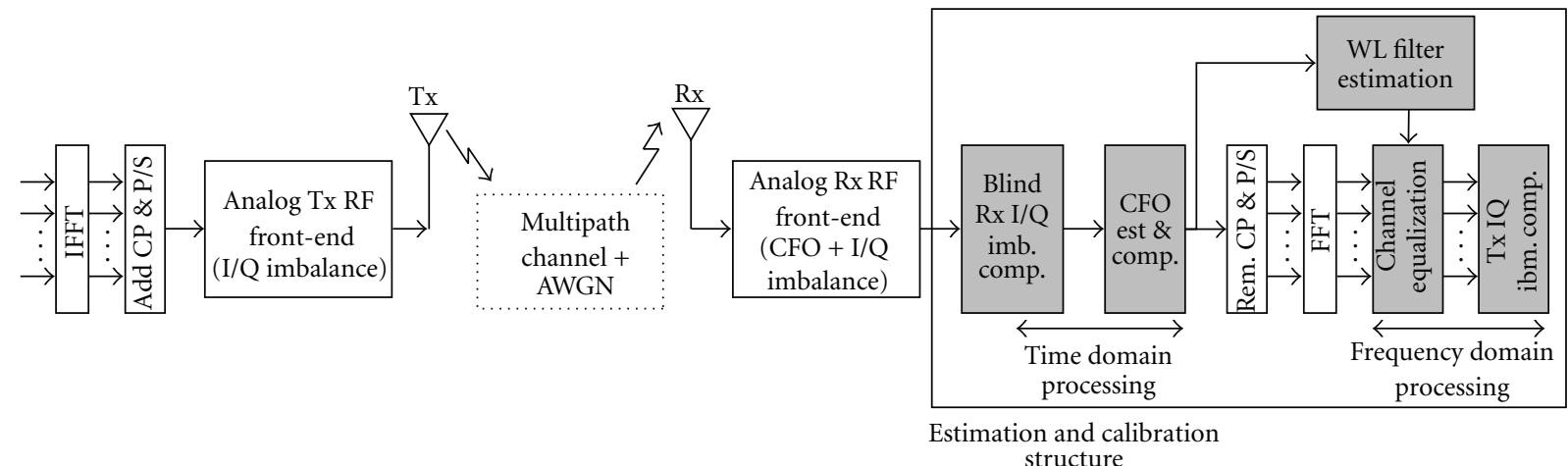

FIGURE 6: OFDM system model with sparse pilot compensator structure.

(6) From above equation, we find the compensation filter at the pilot subcarriers as

$$
W_{k}^{p}=\frac{U_{k}^{p}-\widetilde{S}_{k}^{p}}{\breve{S}_{-k}^{p}} .
$$

(7) Interpolate $W_{k}^{p}$ to all subcarriers to obtain $W_{k}$ (see Section 3.3.4).

(8) The estimated symbols are then given as

$$
\widehat{U}_{k}^{i}=\widetilde{S}_{k}^{i}+W_{k} \widetilde{S}_{-k}^{i} .
$$

3.3.3. Estimation of Widely Linear Filter $G_{D, K}$. The filter $G_{D, k}$ can also be estimated in the time domain as discussed in the previous subsection. For the $i$ th received OFDM symbol after receiver I/Q imbalance and CFO compensation, we perform the DFT operation and pick the data on pilot subcarriers. The time domain OFDM symbol corresponding to pilot subcarriers can then be written as $\overline{\widetilde{\mathbf{y}}}^{p}(n) \triangleq$ $\left[\tilde{y}^{p}(n), \tilde{y}^{p}(n-1), \ldots, \tilde{y}^{p}\left(n-N_{p}+1\right)\right]^{T}$ and the observable imbalance filter as $\overline{\mathbf{g}}_{D}=\left[\widehat{g}_{D, 1}, \widehat{g}_{D, 2}, \ldots, \widehat{g}_{D, N_{\sigma}}\right]^{T}$, where again $N_{p}$ is the length of sparse pilot symbols and $N_{g}<L_{\mathrm{CP}}$ is the length of estimated filters. The LS estimate of $\overline{\mathbf{g}}_{D}$ is then

$$
\overline{\mathbf{g}}_{D}=\left(\overline{\mathbf{U}}^{p H}(n) \overline{\mathbf{U}}^{p}(n)\right)^{-1} \overline{\mathbf{U}}^{p H}(n) \overline{\widetilde{\mathbf{y}}}^{p}(n),
$$

where $\overline{\mathbf{U}}_{p}(n)$ is the time-domain circular-convolution matrix formed from by taking the IDFT of pilot symbols $\mathbf{u}_{p}$.

3.3.4. Interpolation of $W_{k}^{p}$. Here, we derive the transformation for the interpolation of filter $W_{k}^{p}$ in (30). Assume that the pilot estimates stem from a time domain impulse response $\overline{\mathbf{w}}$ of length $N_{w}$, then the model expressed in (30) becomes

$$
\mathbf{W}^{p}=\mathbf{F}_{\left\{\operatorname{ind} P, 1: N_{w}\right\}} \overline{\mathbf{w}},
$$

where $\mathbf{W}^{p}$ is $N_{p} \times N_{p}$ diagonal matrix and ind $P$ denotes the indices of pilot subcarriers. Now, let us define the DFT matrix $\mathbf{F}_{M}$ comprised of first $N_{w}$ columns and rows corresponding to the pilot subcarriers, formally given as

$$
\mathbf{F}_{M}=\mathbf{F}_{\left\{\operatorname{ind} P, 1: N_{w}\right\}},
$$

then the impulse response vector is estimated based on minimizing the mean square error

$$
\arg \min _{\overline{\mathbf{w}}}\left\|\mathbf{W}^{p}-\mathbf{F}_{M} \overline{\mathbf{w}}\right\|^{2}
$$

which can be found by minimizing the LS scheme, corresponding to left multiplying th pseudo-inverse of $\mathbf{F}_{M}$ to (30) yielding

$$
\overline{\mathbf{w}}_{\mathrm{LS}}=\left(\mathbf{F}_{M}^{H} \mathbf{F}_{M}\right)^{-1} \mathbf{F}_{M}^{H} \mathbf{W}^{p} .
$$

The interpolated frequency-domain coefficients are then obtained as

$$
\mathbf{W}_{\mathrm{LS}}=\mathbf{F}_{\left\{1: N, 1: N_{w}\right\}} \overline{\mathbf{W}}_{\mathrm{LS}} .
$$

The estimates obtained from the above equation are then plugged in (31) to obtain transmitted symbols estimate.

In practice, some of the coefficients of $\mathbf{W}_{\mathrm{LS}}$ might be very biased due to deep channel fading. These biased estimates can be improved by using weighted-least-squares (WLS) solution. There are many possibilities for defining the weight matrix, for example, giving more weight to the subcarriers that have strong mirror subcarrier. In this work, we define the weight matrix as

$$
\mathbf{P}_{k}=\operatorname{diag}\left\{\frac{\left|\hat{U}_{-k}^{*}\right|^{2}}{\left|\hat{U}_{k}\right|^{2}}\right\},
$$

which transform the original LS problem into WLS as

$$
\overline{\mathbf{w}}_{\mathrm{WLS}}=\left(\mathbf{F}_{M}^{H} \mathbf{P} \mathbf{F}_{M}\right)^{-1} \mathbf{F}_{M}^{H} \mathbf{P} \mathbf{W}^{p}
$$

or equivalently in frequency domain as

$$
\mathbf{W}_{\mathrm{WLS}}=\mathbf{F}_{\left\{1: \mathrm{N}, 1: N_{w}\right\}} \overline{\mathbf{W}}_{\mathrm{WLS}} .
$$

3.4. Compensation Complexity. In Table 1, we summarize the complexity in terms of number of the complex multiplications required by each compensation stage.

The overall complexity of the proposed compensation schemes is $\left(N_{w}+1\right) \times\left(N+L_{\mathrm{CP}}\right)+2 N_{a}$. 
TABle 1: Computational complexities per OFDM symbol for different compensation stages.

\begin{tabular}{lc}
\hline Algorithms & Complex multiplications \\
\hline Rx. I/Q imbalance compensation & $N_{w} \times\left(N+L_{\mathrm{CP}}\right)$ \\
CFO compensation & $N+L_{\mathrm{CP}}$ \\
Channel equalization & $N_{a}$ \\
Tx. I/Q imbalance compensation & $N_{a}$ \\
\hline
\end{tabular}

Conceptually, a conventional channel equalizer in the absence of CFO and I/Q imbalances operates on a persubcarrier basis, requiring 1 complex multiplication per active subcarrier. However, a joint equalizer for channel, CFO, and Tx. Rx. imbalances has to take into account the contribution of all active subcarriers. Therefore, for such an equalizer with $N_{a}$ active subcarriers, $N_{a}^{2}$ complex multiplications would be required. Comparing this complexity with the complexity of proposed decoupled schemes clearly signifies the benefit of decoupled compensation.

\section{Simulation Results}

The performance of proposed algorithms is illustrated in this section with computer simulations. We first evaluate the performance of CFO estimator in the presence of transmitter I/Q imbalance and channel distortions and do not take into account receiver I/Q imbalance, for both preamble and sparse pilot structures. Then, SER simulations are performed to evaluate the detection performance of compensation schemes in the presence of transmitter and receiver I/Q imbalance, channel distortions, and CFO.

4.1. Performance of CFO Estimator. The parameters considered for CFO estimator simulations are as follows: OFDMbased system with 64-QAM subcarrier modulation, total number of subcarriers $N=1024$, of which 600 are active, 50 OFDM symbols, and the subcarrier spacing is $15 \mathrm{KHz}$. In the case of preamble pilot, the first complete OFDM symbol is considered as pilot and used for the CFO estimation. For sparse pilot example, pilot symbols are inserted at every sixth subcarrier. The multipath channel is 39 taps long with maximum delay spread of $2.5 \mathrm{~ms}$ and power delay profile as described in [34]. The transmitter I/Q imbalance filters impulse responses are $\overline{\mathbf{h}}_{I ; T}=[1,0,0]^{T} ; \overline{\mathbf{h}}_{Q ; T}=$ $[0.97,-0.1,0.01]^{T}$. Frequency-independent gain $g_{T}$ and phase $\phi_{T}$ are chosen corresponding to IRR levels of $20 \mathrm{~dB}$, $30 \mathrm{~dB}, 40 \mathrm{~dB}$, and infinite (INF.). These IRR levels map to gain imbalance of $10 \%, 3 \%, 1 \%, 0 \%$ and phase imbalance of $10^{\circ}, 4^{\circ}, 1^{\circ}, 0^{\circ}$, respectively. The mean received SNR is $20 \mathrm{~dB}$. The performance results are illustrated by plotting the mean square error (MSE) over normalized CFO values with 500 independent channel realizations: $\mathrm{MSE}=E|\Delta \hat{f}-\Delta f|^{2}$

Figures 7 and 8 show the performance of CFO estimation scheme of [25] for preamble and sparse pilot cases, respectively. For the sparse pilot example, we assume that the system under consideration is LTE for which normalized CFO can be higher than one [25], and we simulate the algorithm over the normalized CFO range of $[-5,5]$. As

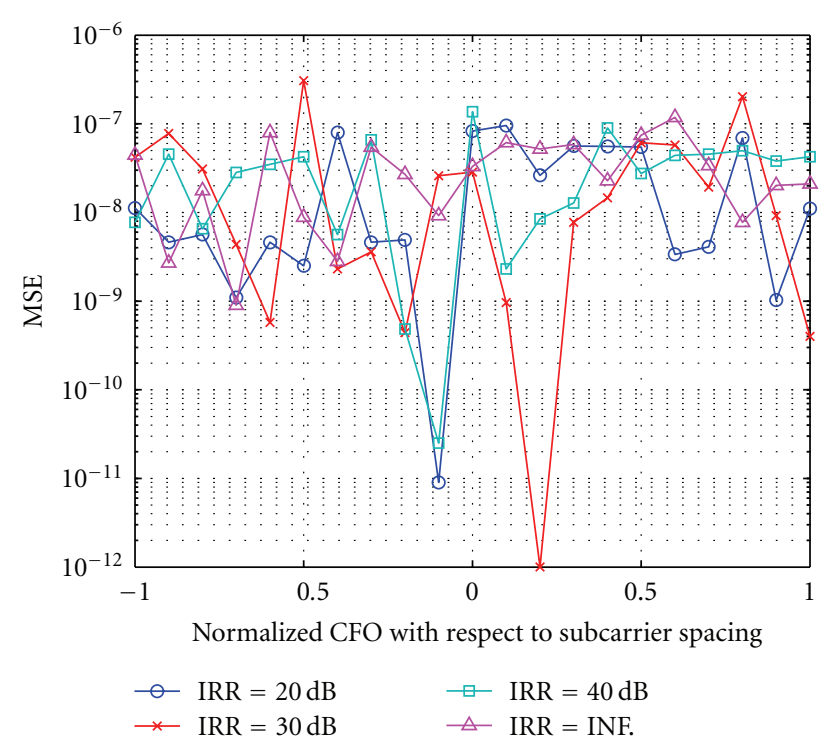

Figure 7: MSE of preamble pilot-based CFO estimator versus normalized CFO for 64-QAM OFDM syste; 39-tap veh. A channel model; ensemble average of 500 independent channel realizations.

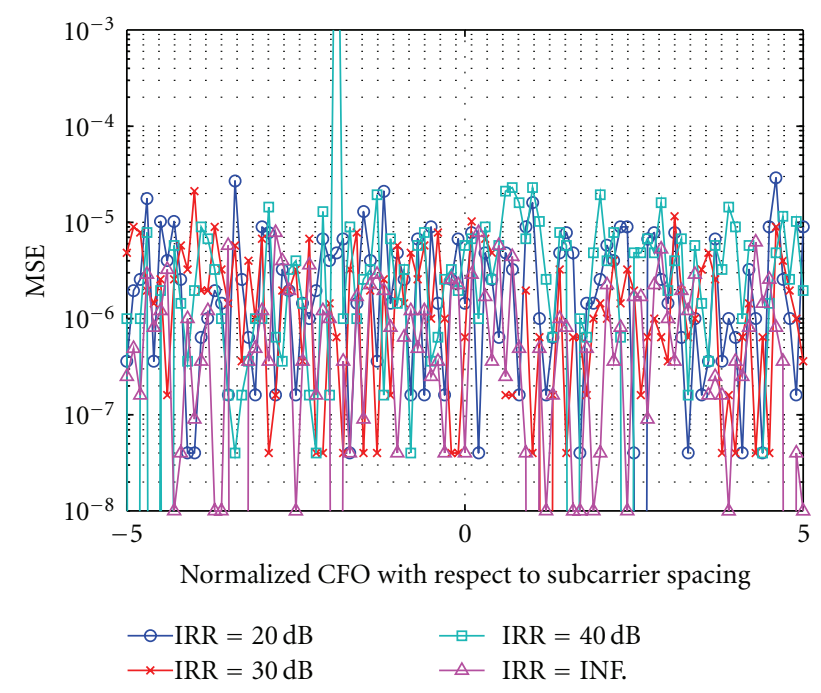

FIGURE 8: MSE of sparse pilot-based CFO estimator versus normalized CFO for 64-QAM OFDM syste; 39-tap veh. A channel model; ensemble average of 500 independent channel realizations.

evident from the results, the algorithm delivers sufficiently reliable estimates for small to large imbalance values. The MSE remains below than $10^{-7}$ with preamble and in the order of $10^{-5}$ when pilots are inserted sparsely at SNR = $20 \mathrm{~dB}$.

4.2. SER Performance. Now, we perform the SER simulations and illustrate the results by plotting the mean SER as a function of received SNR. A typical 64-QAM OFDM system is considered whose parameters are given in Table 2. The OFDM signal parameters are similar to 3GPP longterm evolution (LTE) specifications [35]. The I/Q imbalance 
TABLE 2: System parameters.

\begin{tabular}{lc}
\hline Total number of subcarriers & $N=1024$ \\
Active subcarriers & $N_{a}=600$ \\
Cyclic prefix length & $L_{\mathrm{CP}}=52$ \\
Subcarrier spacing & $1 / N T_{s}=15 \mathrm{KHz}$ \\
Sampling Frequency & $F_{s}=15.36 \mathrm{MHz}$ \\
Channel type & vehicular A \\
No. of channel taps & 39 \\
Gain imbalance & $g_{T ; R}=5 \%$ \\
Phase imbalance & $\phi_{T ; R}=5^{\circ}$ \\
I-branch filter & $\overline{\mathbf{h}}_{I, T ; R}=[1,0,0]^{T}$ \\
Q-branch filter & $\overline{\mathbf{h}}_{\mathrm{Q}, T ; R}=[0.97,-0.05,0.01]^{T}$ \\
\hline
\end{tabular}

values stated in the table correspond to $23-30 \mathrm{~dB}$ front-end IRR and represent a practical example case.

In the following figures, legend "No FE Distortion" refers to the case when no transmitter and receiver I/Q imbalance and CFO is present and known channel estimates are used for channel equalization, "W/o Compensation" refers to when all radio impairments are present and only channel is equalized with known estimates, and "W/Tx Rx IQ Imbalance only" for the case when only transmitter and receiver IQ imbalance is present and channel is equalized with known estimates. "W/ZF Compensation" "W/ML Compensation" "W/LS Compensation", and "W/WLS Compensation" legends exemplify the schemes proposed in Section 3. The results depicted are averaged over $10^{3}$ independent channel realizations.

For preamble pilot-based compensation, we transmit 50 OFDM symbols and use first OFDM symbol as pilot. In each simulation run, we generate a random CFO in the range $[-1,1]$ and introduce it to the signal (with transmitter $\mathrm{I} / \mathrm{Q}$ imbalance and channel distortions). The receiver I/Q imbalance is compensated with 3-tap compensation filter, built by utilizing the second-order statistics of the received signal. This is followed by CFO estimation and compensation, performed in time domain. ZF and ML estimation techniques are applied next. It can be seen in Figure 9 that communication system becomes completely unusable when no compensation scheme is in place and SER remains very high when only I/Q imbalance is present at both transmitter and receiver. The ZF equalizer is able to reduce the SER to the level of a system with no front-end distortion. On the other hand, the ML equalizer even outperforms the SER performance of system with no RF impairments and known channel estimates. In the low SNR region, the SER is close to the ideal system but as the SNR increases, the equalizer is able to extract the frequency diversity gain induced by transmitter I/Q imbalance. A similar diversity effect is also reported in [29] where only transmitter I/Q imbalance and channel distortions are considered.

The OFDM signal model for sparse pilot-based compensation is similar to LTE frame structure shown in Figure 10, consisting of one frame that is composed of ten subframes; each subframe is further divided into two slot. A slot consists of seven OFDM symbols, and pilot symbols are

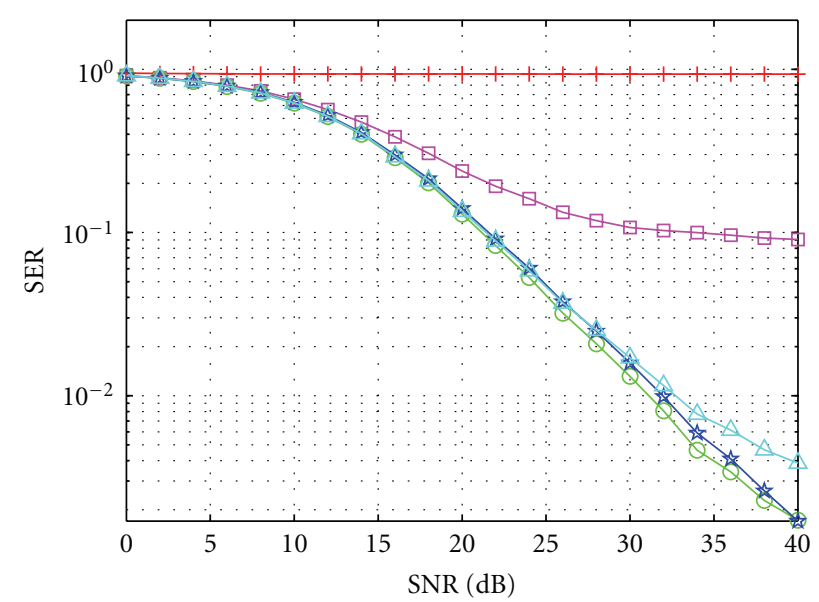

$\begin{array}{ll}\rightarrow \text { W/o compensation } & - \text { No FE distortion } \\ \square-\text { W/Tx Rx IQ imbalance only } & \triangle \text { W/ZF compensation } \\ - & \end{array}$

FIGURE 9: Preamble pilot-based SER versus SNR performance curves for 64-QAM OFDM system. Tx/Rx FE IRR = 20-30 dB; veh. A channel model with 39 taps; ensemble average over 1000 independent channel realizations.

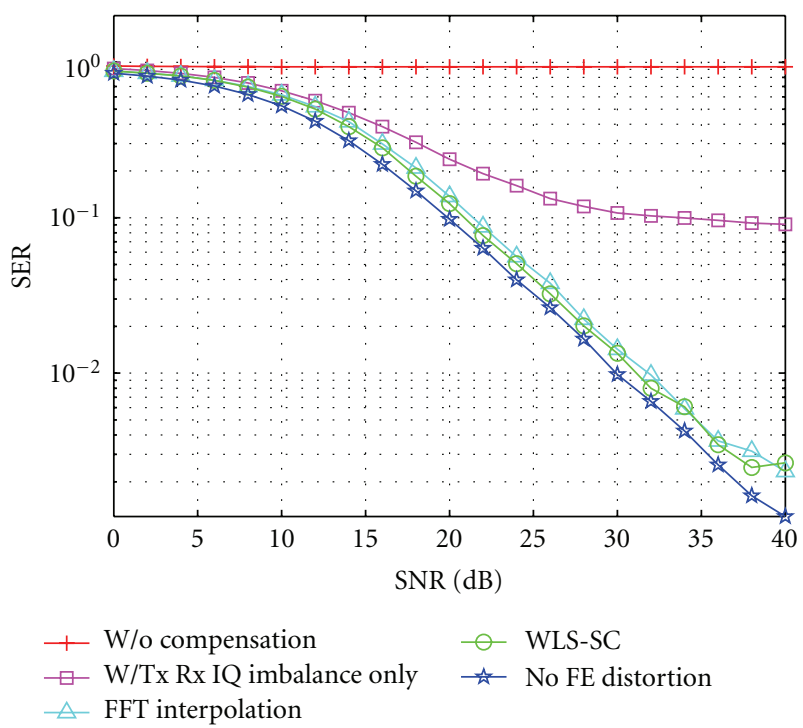

FIGURE 10: Sparse pilot-based SER versus SNR performance curves for 64-QAM OFDM system. Tx/Rx FE IRR = 20-30 dB; veh. A channel model with 39 taps; ensemble average over 1000 independent channel realizations.

located at every sixth subcarrier of first and fifth OFDM symbol of each slot. Thus, in total, we transmit 140 OFDM symbols with pilot tones on 20 symbols. As discussed in the previous subsection, the normalized frequency offset is randomly generated in the range $[-5,5]$ during each channel realization (CFO in LTE is much smaller, in fact it can be greater than one), and its effect is added to the signal. At the algorithm compensation level, we again apply a 3-tap compensation filter for receiver I/Q compensation, followed by CFO estimation and compensation. The LS and WLS compensation algorithms are applied in frequency 


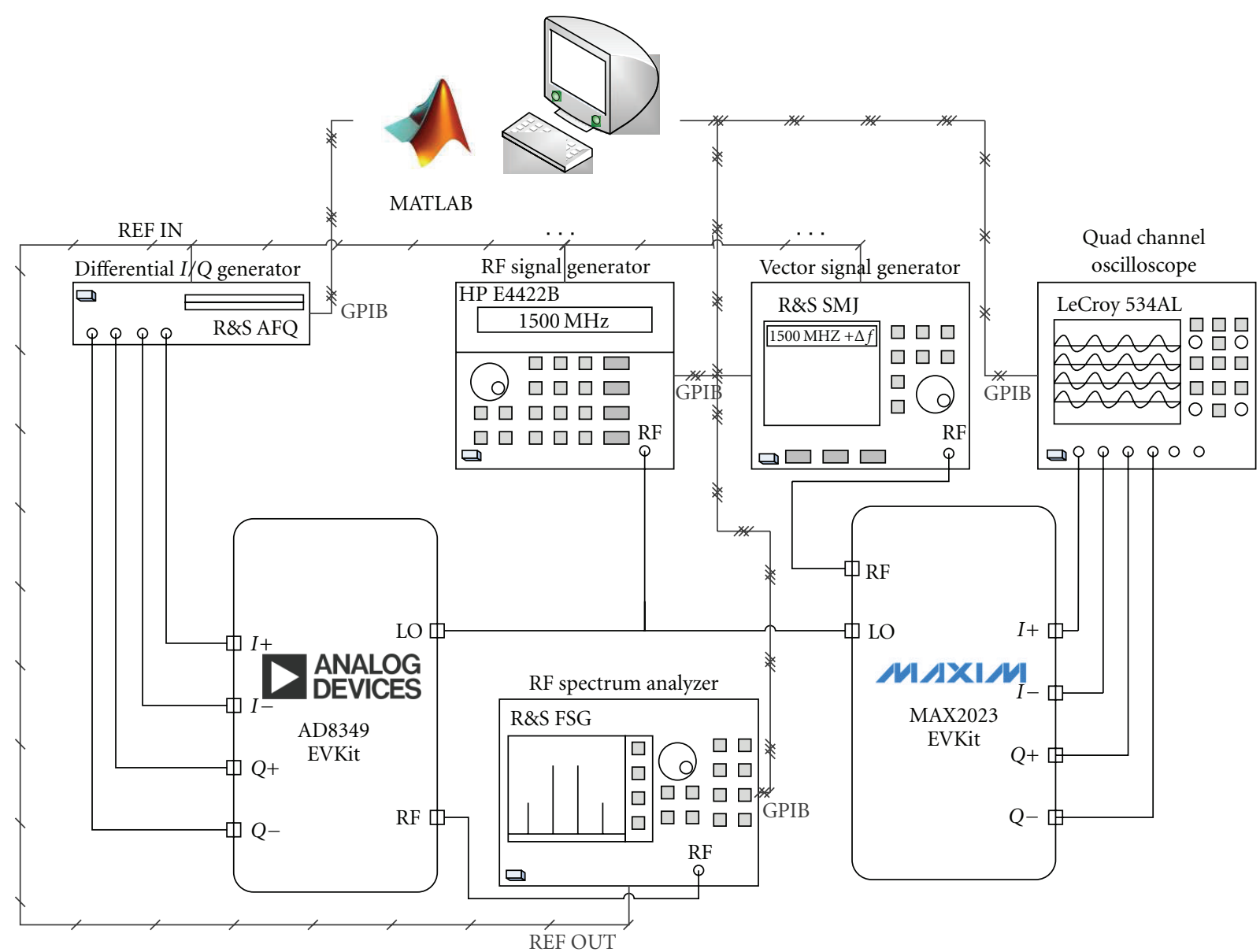

FIGURE 11: General structure of the measurement setup consisting of laboratory instruments and I/Q modulator and demodulator.

domain for channel distortions and transmitter I/Q imbalance calibration. Figure 10 illustrates the simulation results that renders excellent calibration property of proposed algorithms, with WLS achieving close to SER of a system with no FE distortions.

\section{Experimental RF Measurements Results}

In order to further validate the performance of proposed compensation algorithms, real-world RF measurements have been performed. We first summarize the overall measurement setup and then describe the measurement procedure in detail.

5.1. Prototype Implementation. The generic structure of the measurement setup is identical to the one shown in Figures 4 and 6 , and the corresponding block diagram is depicted in Figure 11. The prototype implementation integrates Rohde and Schwarz $(\mathrm{R} \& S)$ AFQ100A baseband I/Q signal generator, R\&S FSG spectrum and signal analyzer, R\&S SMJ vector signal generator, HP E4422B RF signal generator, LeCroy 534AL quad-channel oscilloscope, Analog Devices (AD) AD8349 direct conversion modulator evaluation kit, and Maxim 2023 demodulator evaluation kit. All the instruments are connected to a computer via general purpose interface bus (GPIB) for remote control operation.
5.2. Measurement Setup. A computer with MATLAB is used for performing digital signal processing-related tasks and also for instrument control. The baseband OFDM signal with parameters similar to simulations as in Section 4 is generated in computer, and its samples are loaded into the memory of R\&S AFQ. The analog I/Q signal output by R\&S AFQ is upconverted to RF frequency by AD8349 modulator. In order to introduce the channel distortions and noise, we capture the baseband equivalent of the RF signal using built-in $I / Q$ subsystem of R\&S FSG. The I/Q subsystem first translates the signal to an intermediate frequency (IF) of $20.4 \mathrm{MHz}$, followed by IF filtering. The filtered signal is then digitized by a built-in ADC at a rate of $81.6 \mathrm{MHz}$. Finally the sampled signal is digitally downconverted to complex baseband, lowpass filtered, and resampled to desired sampling frequency. The samples are then read out in block of 512,000 each in ASCII format. The channel distortions and noise are then added, and the samples of signal (with transmitter I/Q imbalance, channel distortions, and noise) are loaded into R\&S SMJ memory, which outputs the RF signal. The CFO effect is introduced at the transmitter side by drifting the LO frequency of R\&S SMJ relative to the RF frequency of HP E4422B. The LO frequency is used in the measurements in $1.5 \mathrm{GHz}$. On the receiver side, MAX2023 demodulator downconverts the signal from RF to baseband. 
The output of analog frontend of MAX2023 is captured with a quad channel oscilloscope. The samples recorded by the oscilloscope are then sent to MATLAB for offline processing.

\subsection{Performance Analysis}

5.3.1. Front-End IRR without Calibration. We first shortly address the estimation of front-end IRR of AD8349 and MAX2023 chips, without any compensation. Although the exact values of gain and phase imbalance of these chips are unknown, the IRR analysis is still possible using LS fitting approach as follows. We denote the reference time domain signal $\overline{\mathbf{z}}$ to be transmitted. The complex envelope of RF signal can be written as in (41). $\overline{\mathbf{Z}}(n)$ and $\overline{\mathbf{Z}}^{*}(n)$ are time domain convolution matrix and conjugate convolution matrix formed from reference signal $\overline{\mathbf{z}}(n)$ and its conjugate $\overline{\mathbf{z}}^{*}(n)$, respectively. Assuming perfect synchronization in time, frequency, and phase, the LS estimates of the front-end imbalance filters are then given by $(42)[8,11]$.

In Figure 12, IRR curves with different I/Q imbalance filter lengths, utilizing 50,000 samples and ensemble averaged over ten independent simulation runs, are plotted. The LO frequency is $1.5 \mathrm{GHz}$, and the signal is a 16-QAM modulated signal with $18 \mathrm{MHz}$ two-sided bandwidth, six times oversampling, and root raised cosine pulse shaping. The results suggest that both chips have frequency selective image rejection, varying between $27-29 \mathrm{~dB}$ for $\mathrm{AD} 8349$ (transmitter modulator) and 30-33 dB for Maxim 2023 (receiver demodulator):

$$
\begin{aligned}
\overline{\mathbf{s}}(n)= & {\left[\begin{array}{c}
s(n) \\
s(n-1) \\
\vdots \\
s(n-L+1)
\end{array}\right] } \\
= & {\left[\begin{array}{c}
\overline{\mathbf{g}}_{1, T}^{T}(n) \overline{\mathbf{z}}(n)+\overline{\mathbf{g}}_{2, T}^{T}(n) \overline{\mathbf{z}}^{*}(n) \\
\overline{\mathbf{g}}_{1, T}^{T}(n) \overline{\mathbf{z}}(n-1)+\overline{\mathbf{g}}_{2, T}^{T}(n) \overline{\mathbf{z}}^{*}(n-1) \\
\vdots \\
\overline{\mathbf{g}}_{1, T}^{T}(n) \overline{\mathbf{z}}(n-L+1)+\overline{\mathbf{g}}_{2, T}^{T}(n) \overline{\mathbf{z}}^{*}(n-L+1)
\end{array}\right] } \\
= & {\left[\overline{\mathbf{Z}}(n) \overline{\mathbf{Z}}^{*}(n)\right]\left[\begin{array}{c}
\overline{\mathbf{g}}_{1, T}(n) \\
\overline{\mathbf{g}}_{2, T}(n)
\end{array}\right]=\overline{\mathbf{Z}}_{b}(n)\left[\begin{array}{l}
\overline{\mathbf{g}}_{1, T}(n) \\
\overline{\mathbf{g}}_{2, T}(n)
\end{array}\right], } \\
{\left[\begin{array}{c}
\overline{\mathbf{g}}_{1, T}(n) \\
\overline{\mathbf{g}}_{2, T}(n)
\end{array}\right]=} & \overline{\mathbf{Z}}_{b}^{\dagger}(n) \overline{\mathbf{s}}(n) .
\end{aligned}
$$

5.3.2. Blind Receiver I/Q Imbalance Compensation. The performance of blind receiver I/Q imbalance compensation algorithm is evaluated next. Here, R\&S SMJ acts as transmitter to produce RF signal (with no transmitter IQ imbalance and channel distortions) and MAX2023 to demodulate the signal. The digitized baseband signal (with receiver I/Q imbalance) is transferred to computer through GPIB, where we apply blind DSP algorithm for compensation. The data captured from oscilloscope, however, cannot be used directly for compensation but rather it is first processed to remove the RF chain errors. That involves resampling, delay removal, phase synchronization, offset cancellation, and scaling. After

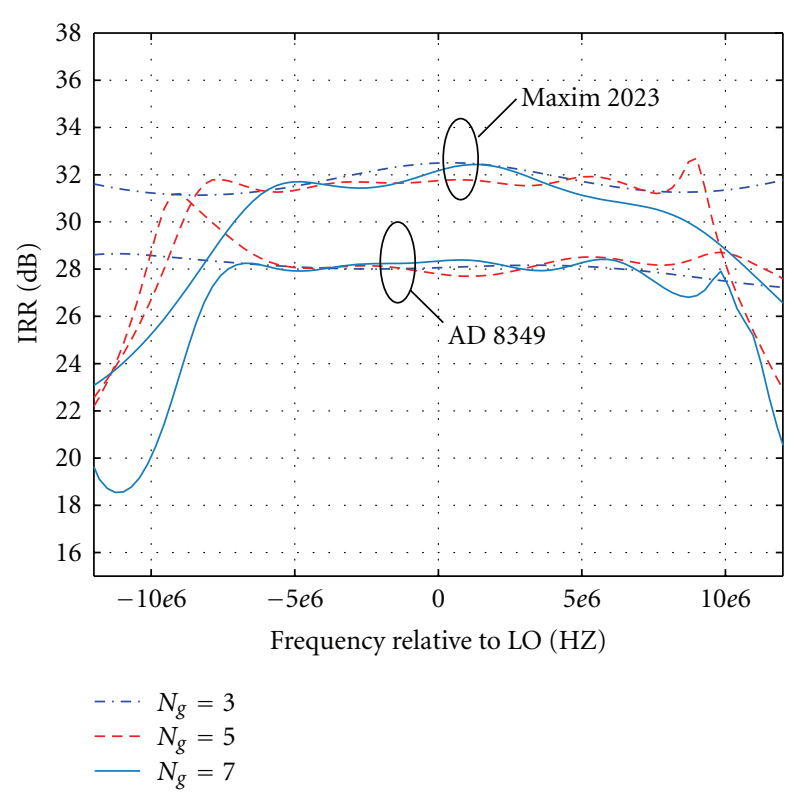

FIGURE 12: Comparison of averaged FE IRR versus frequency for different imbalance filter lengths. $\mathrm{LO}$ frequency $=1.5 \mathrm{GHz}$.

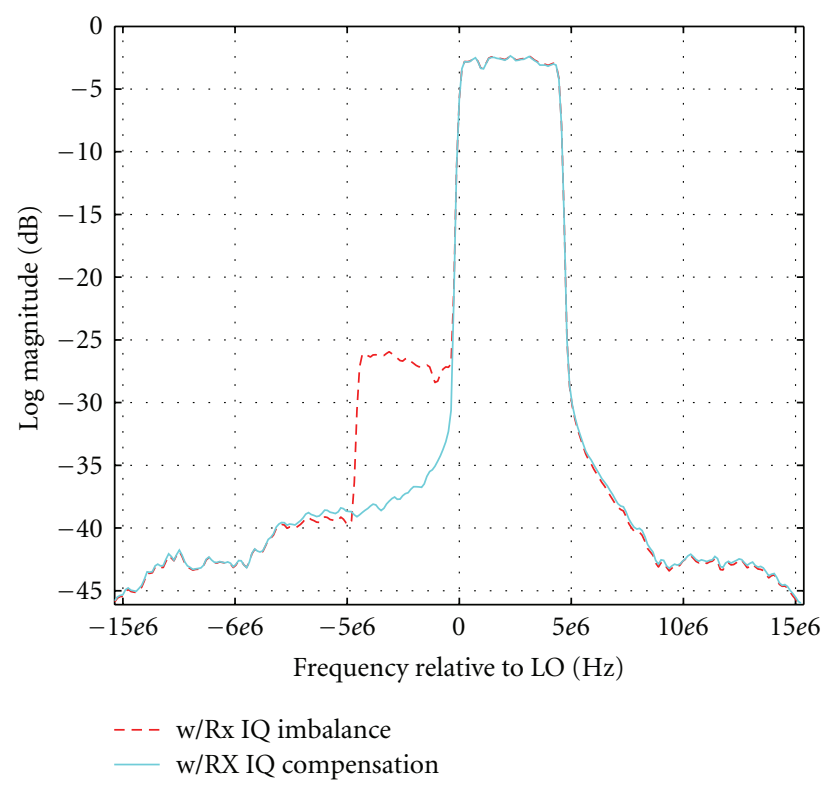

FIGURE 13: Comparison of measured I/Q demodulator output before and after I/Q imbalance compensation.

these steps, we apply the compensation algorithm. For illustration purpose, we use a half-loaded OFDM signal with 300 active subcarriers (out of total 1024). The LO frequency is $1.5 \mathrm{GHz}$. Figure 13 shows the measured spectra before and after compensation, evidencing a clear improvement obtained with the compensation algorithm.

5.3.3. Characterization of Pilot-Based Compensation Schemes. The performance analysis of pilot-based compensation schemes is carried out by creating an OFDM waveform with parameters given in Table 2 as per LTE standard. The 


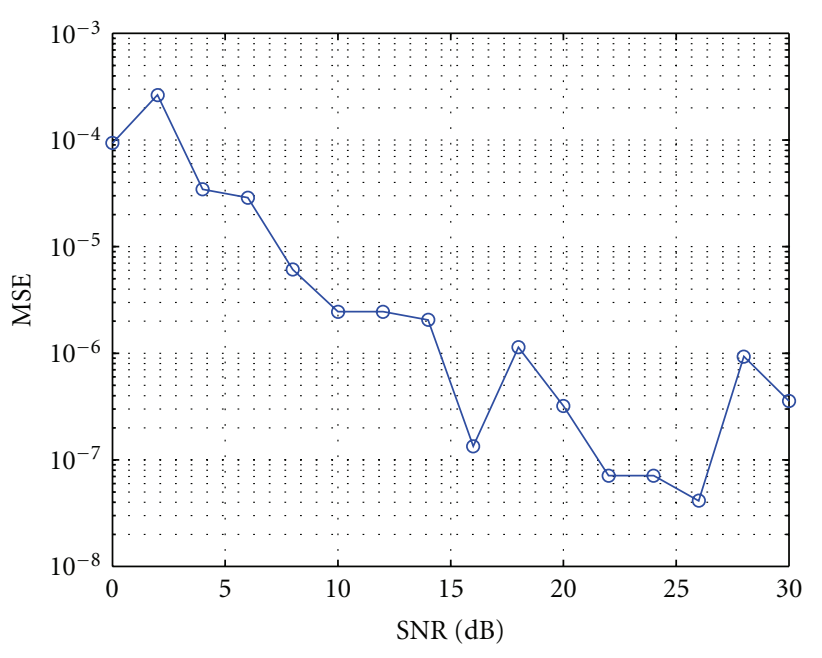

FIgure 14: Preamble pilot-based measured MSE of CFO versus SNR performance curve. $\mathrm{CFO}=10 \mathrm{KHz}$.

baseband OFDM signal is sampled at $2 \times 1024 \times 15 \mathrm{KHz}=$ $30.72 \mathrm{MHz}$, oversampled by 2 , and output by R\&S AFQ. The I/Q subsystem of R\&S FSG implements the down conversion, filtering, and sampling from $81.6 \mathrm{MHz}$ to $2 \times$ $30.72 \mathrm{MHz}=61.44 \mathrm{MHz}$. Blocks of 512,000 samples are read from R\&S FSG memory and loaded into MATLAB memory. The samples are processed for delay estimation, timing synchronization, and LO leakage removal. After the data conditioning, the channel distortions and noise are added, before feeding the signal to R\&S SMJ. The CFO effect is also introduced in R\&S SMJ by changing the RF frequency. The RF signal is downconverted and sampled at $50 \mathrm{M}$ Samples/second. These samples are extracted from oscilloscope and again processed in MATLAB for resampling to $30.72 \mathrm{MHz}$, delay estimation, phase synchronization, and scaling. After these processing stages, we have the baseband equivalent model of the signal that has transmitter/receiver I/Q imbalance, channel distortion, noise, and CFO effects included.

The example waveform used for preamble pilot-based compensation algorithms is an OFDM signal with same parameters as were in simulations. In the measurements, we transmit 10 OFDM symbols and use one known OFDM symbol during the estimation and calibration phase. The CFO is $10 \mathrm{KHz}$ and a 3-tap compensation filter for receiver I/Q imbalance compensation and also for transmitter I/Q imbalance estimation. Both ML and ZF equalization schemes are applied to the signal, after receiver I/Q imbalance and CFO compensation. With a fixed channel, the obtained results ensemble averaged over 10 independent measurements are plotted. Figure 14 shows the measurement results of CFO estimation over different SNR values. The results indicate that the estimator performance improves with increasing SNR. Even for small SNR, the MSE remains very low. In Figure 15, measured SER versus SNR is plotted. The figure also shows a reference curve ("No FE Distortion,") obtained with MATLAB simulation with no I/Q imbalances, perfect $\mathrm{CFO}$, and channel compensation with known

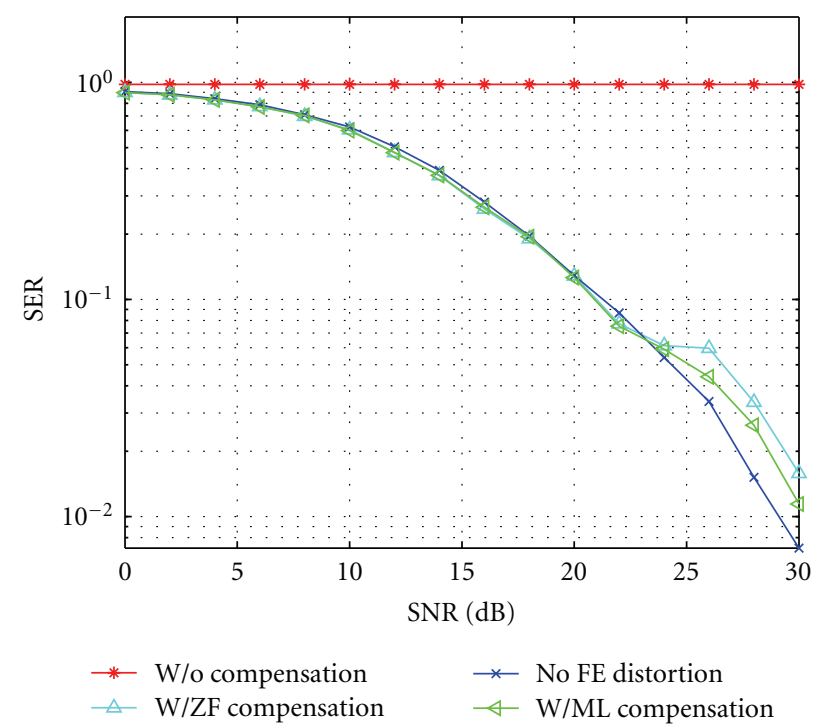

FIGURE 15: Measured SER versus SNR performance curves for 64QAM OFDM system with preamble pilot; $\mathrm{CFO}=10 \mathrm{KHz}$; veh. A channel model with 39 taps; ensemble average over 10 realizations with a fixed channel.

estimates. As evident from Figure 15, the algorithms give very consistent performance with SER close to simulated SER. Also, the ML technique provides better performance than ZF technique. Notice that for SNR greater than 26, there is a little performance degradation which is most likely due to measurement noise. In Figure 16, we also plot the symbol constellation after compensation with ZF equalization scheme. The excellent calibration property of algorithms is also visible here.

In the second measurement example, the signal is an OFDM signal having 10 symbols and pilots located at every sixth subcarrier. The signal has altogether 600 active subcarriers out of 1024 subcarriers, with $15 \mathrm{KHz}$ subcarrier spacing. Therefore, in total, there are 1000 pilot symbols. The signal is oversampled by 2 . The CFO is now $45 \mathrm{KHz}$ that is greater than the subcarrier spacing. Again the receiver I/Q imbalance is compensated with 3-taps, and the LS and WLS approaches discussed in Section 3 are employed for transmitter I/Q imbalance and channel equalization. The CFO estimation in terms of MSE between the estimated and the true CFO value versus SNR is plotted in Figure 17, which demonstrates a similar behavior to previous preamble pilot-based estimation example. The measured SER versus SNR curve in Figure 18 and symbols after compensation are plotted in Figure 19. The results suggest that the proposed methods are able to deliver very accurate estimates. An important implication of Figure 18 is that with more pilot data available over different symbols, we are able to reduce the effect of measurement noise which was very dominant in the previous measurement example (Figure 15).

In general, it can be concluded from the measurements results of Figures 15 and 18 that the proposed estimator and compensator structures provide considerable improvements to achievable SER, giving confidence to deploy them in practical systems. 


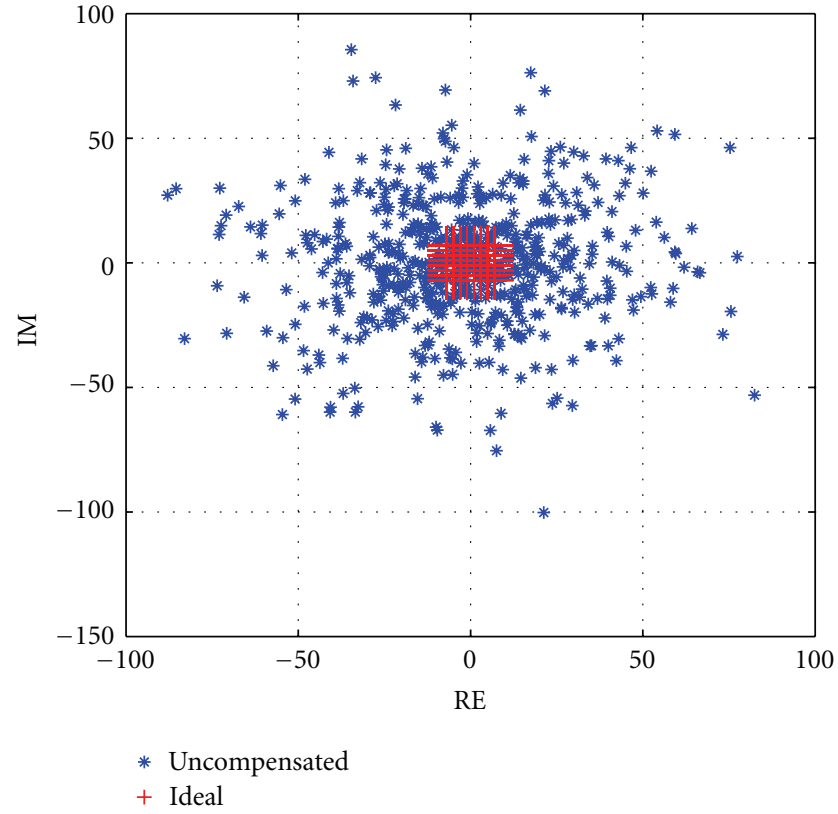

(a)

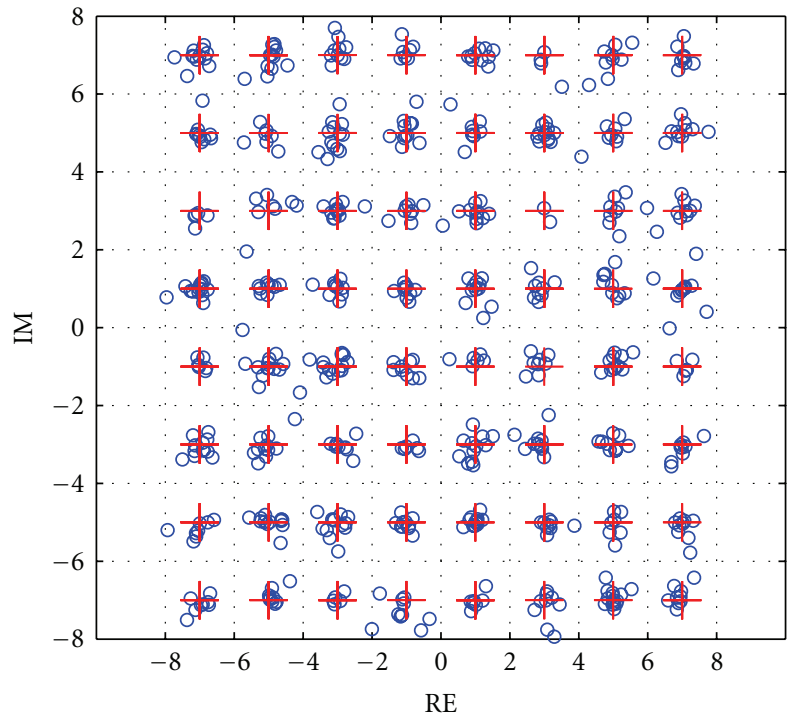

○ W/ZF compensation

+ Ideal

(b)

FIGURE 16: Measured constellation diagram of 64-QAM OFDM system before and after RF impairments mitigation. (a) Uncompensated (b) Compensated with ZF equalization.

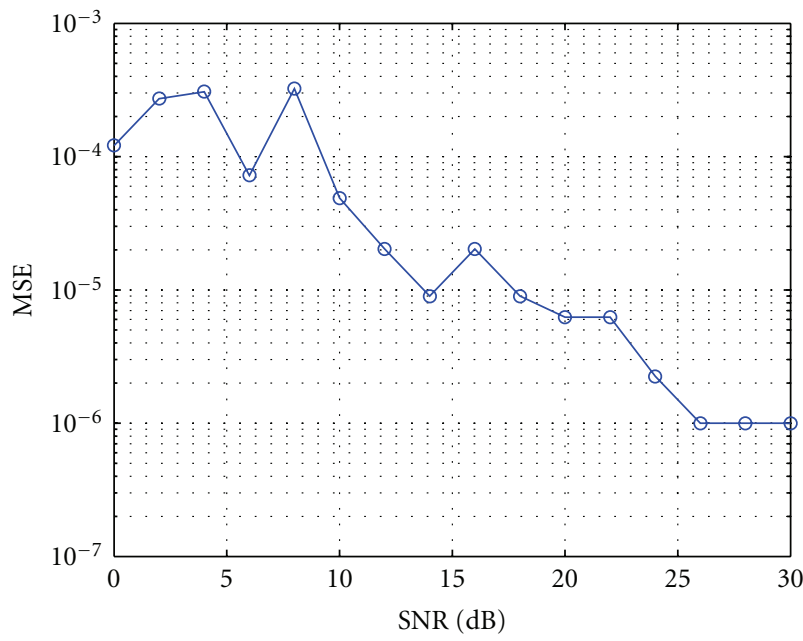

FIGURE 17: Sparse pilot-based measured MSE of CFO versus SNR performance curve. $\mathrm{CFO}=45 \mathrm{KHz}$.

5.4. Implementation Challenges. In practice, the measurement setup is exposed to variety of errors, which must be corrected before introducing the channel distortion and noise as well as before applying the compensation algorithms. We briefly review these errors now.

The downconversion of RF signal (with transmitter I/Q imbalance) using R\&S I/Q subsystem is subject to unavoidable residual errors such as synchronization, LO leakage, delay between original and measured signal, nonlinearities of components, and noise of its own. Frequency synchronization between the instruments is achieved by connecting the instruments to same reference clock of R\&S FSG, as shown

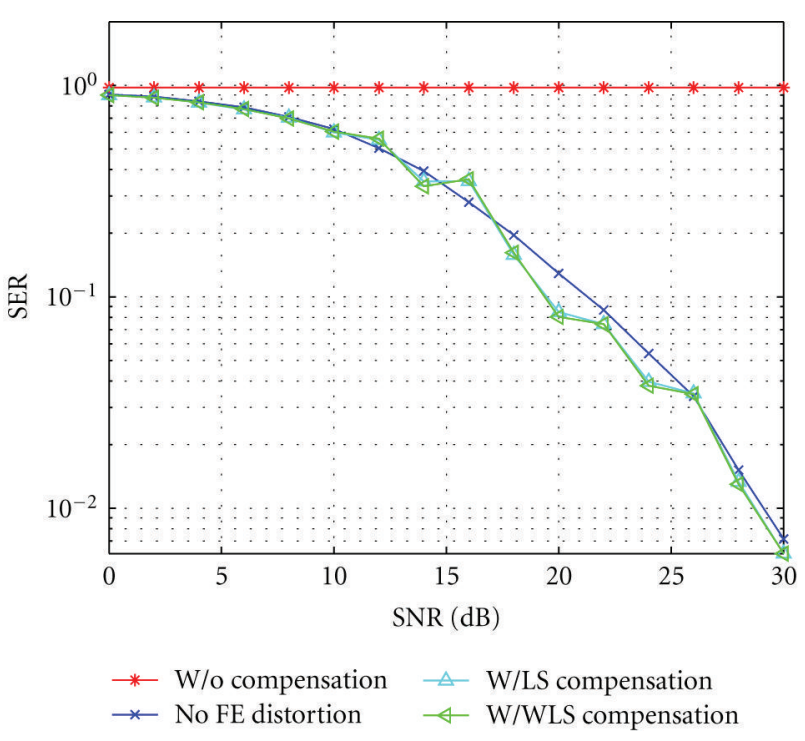

FIGURE 18: Measured SER versus SNR performance curves for 64QAM OFDM system with sparse pilot; $\mathrm{CFO}=45 \mathrm{KHz}$; veh. A channel model with 39 taps; ensemble average over 10 realizations with a fixed channel.

in Figure 11. Symbol timing synchronization is performed prior to downsampling the signal to symbol rate with ML algorithm. LO leakage results due to finite isolation between the LO and RF ports of the modulator/demodulator. Consequently, when RF signal is up-/downconverted, LO leakage causes offset. It can be mitigated by removing the sample mean of the measured signal. The delay estimation is performed in two steps: first integer delay is removed with 


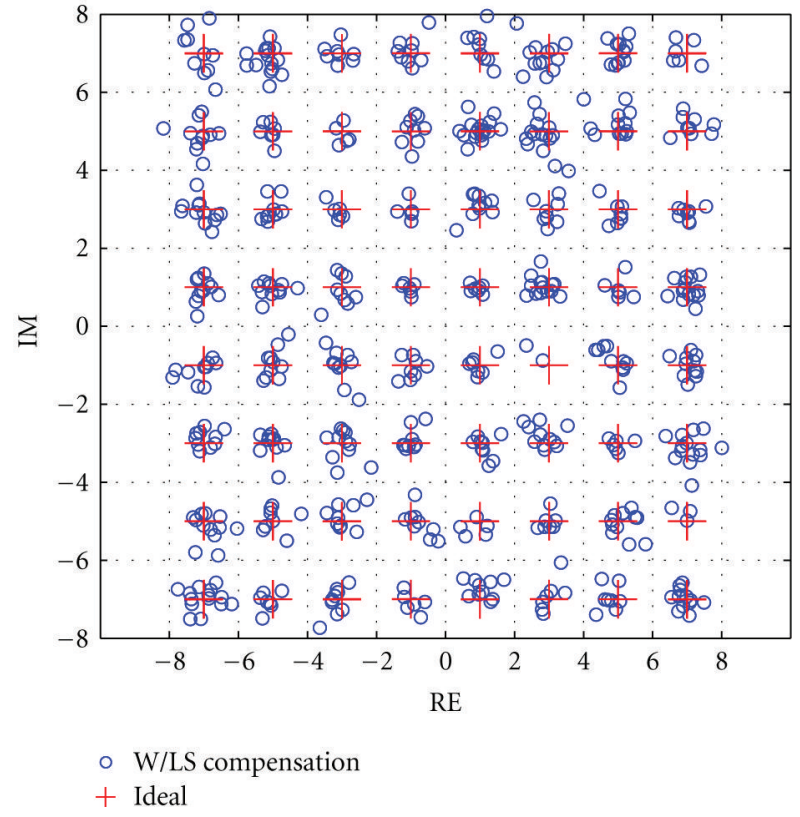

(a)

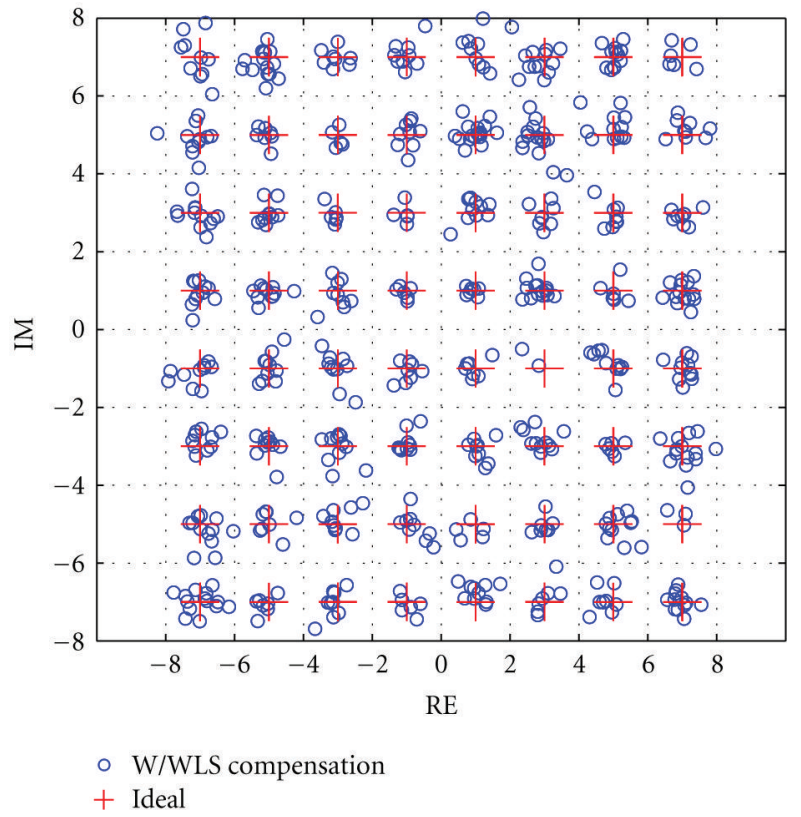

(b)

FIGURE 19: Measured constellation diagram of 64-QAM OFDM system with RF impairments mitigation. (a) Compensated with LS method (b) Compensated with WLS method.

FFT-based correlator, allowing non-data-aided (NDA) ML algorithm for fractional delay estimation and compensation. Detailed review of synchronization algorithms can be found in [36].

Similar to R\&S FSG, the data captured from oscilloscope must be DSP conditioned before being used in actual estimation and compensation. The data conditioning involves resampling to original signal sample rate, delay estimation, and offset removal with already-mentioned algorithms.

\section{Conclusion}

In this paper, the performance of OFDM-based communication systems is studied in the presence of frequency-selective I/Q imbalance, channel distortions and CFO. Based on our signal model, generally applicable algorithms have been developed for the compensation of such impairments. The proposed methods are composed of time domain and frequency domain compensation. It is shown with simulations that the proposed, estimator and compensator structures produce symbol error rates close to the ideal ones. A laboratory measurement setup is also proposed and extensive measurements are carried out to prove the practical value of the algorithms. The measurements results show that the achievable SER is close to the ideal. Hence, the compensation algorithms provide significant improvement to obtainable link performance and can be used in real world radio transceivers. Future work will focus on generalizing the work to MIMO-OFDM and a real-time prototype implementation using FPGA's for the digital parts and integrated RF-ASIC's for the analog circuitry.

\section{Acknowledgments}

This work was supported by the Technology Promotion Foundation of Finland (TES), the Nokia Foundation, the graduate school TISE, the Austrian Center of Competence in Mechatronics (ACCM), the Academy of Finland (under the projects "Digitally-Enhanced RF for Cognitive Radio Devices" and "Joint Analysis and DSP-Based Mitigation of Multiple RF Impairments in Future Radio Devices"), and the Finnish Funding Agency for Technology and Innovation (Tekes; under the project "Enabling Methods for Dynamic Spectrum Access and Cognitive Radio"), all of which are gratefully acknowledged. The authors would also like to thank the anonymous reviewers for their helpful comments and suggestions.

\section{References}

[1] "Wireless LAN medium access control (MAC) and physical layer (PHY) specifications: high speed physical layer in 2.4 GHz band," Tech. Rep., IEEE, 1999.

[2] "IEEE standard for local and metropolitan area networks part 16: air interface for fixed broadband wireless access systems," Tech. Rep., IEEE 802.16, 2004.

[3] U. Ladebusch and C. A. Liss, "Terrestrial DVB (DVB-T): a broadcast technology for stationary portable and mobile use," Proceedings of the IEEE, vol. 94, no. 1, pp. 183-192, 2006.

[4] G. Faria, J. A. Henriksson, E. Stare, and P. Talmola, "DVB-H: digital broadcast services to handheld devices," Proceedings of the IEEE, vol. 94, no. 1, pp. 194-209, 2006.

[5] A. B. Ericsson, "Long term evolution (LTE): an introduction," White Paper, 2007. 
[6] S. Mirabbasi and K. Martin, "Classical and modern receiver architectures," IEEE Communications Magazine, vol. 38, no. 11, pp. 132-139, 2000.

[7] A. A. Abidi, "Direct-conversion radio transceivers for digital communications," IEEE Journal of Solid-State Circuits, vol. 30, no. 12, pp. 1399-1410, 1995.

[8] L. Anttila, M. Valkama, and M. Renfors, "Frequency-selective I/Q mismatch calibration of wideband direct-conversion transmitters," IEEE Transactions on Circuits and Systems II: Express Briefs, vol. 55, no. 4, pp. 359-363, 2008.

[9] L. Ding, Z. Ma, D. R. Morgan, M. Zierdt, and G. T. Zhou, "Compensation of frequency-dependent gain/phase imbalance in predistortion linearization systems," IEEE Transactions on Circuits and Systems I: Regular Papers, vol. 55, no. 1, pp. 390-397, 2008.

[10] M. Valkama, M. Renfors, and V. Koivunen, "Advanced methods for $\mathrm{I} / \mathrm{Q}$ imbalance compensation in communication receivers," IEEE Transactions on Signal Processing, vol. 49, no. 10, pp. 2335-2344, 2001.

[11] L. Anttila, M. Valkama, and M. Renfors, "Circularity-based I/Q imbalance compensation in wideband direct-conversion receivers," IEEE Transactions on Vehicular Technology, vol. 57, no. 4, pp. 2099-2113, 2008.

[12] L. Anttila, M. Valkama, and M. Renfors, "Efficient mitigation of frequency-selective I/Q imbalance in OFDM receivers," in Proceedings of the IEEE Vehicular Technology Conference (VTCF'08), Calgary, Canada, September 2008.

[13] L. Anttila and M. Valkama, "On circularity of receiver frontend signals under RF impairments," in Proceedings of the 17th European Wireless Conference, pp. 610-617, Vienna, Austria, April 2011.

[14] L. Anttila, M. Valkama, and M. Renfors, "Blind moment estimation techniques for I/Q imbalance compensation in quadrature receivers," in Proceedings of the IEEE International Symposium on Personal, Indoor and Mobile Radio Communications (PIMRC '06), Helsinki, Finland, September 2006.

[15] A. Tarighat, R. Bagheri, and A. H. Sayed, "Compensation schemes and performance analysis of IQ imbalances in OFDM receivers," IEEE Transactions on Signal Processing, vol. 53, no. 8, pp. 3257-3268, 2005.

[16] M. Lipardi, D. Mattera, and F. Sterle, "MMSE equalization in presence of transmitter and receiver IQ imbalance," in Proceedings of the International Waveform Diversity and Design Conference (WDD '07), pp. 165-168, Pisa, Italy, June 2007.

[17] A. Tarighat and A. H. Sayed, "Joint compensation of transmitter and receiver impairments in OFDM systems," IEEE Transactions on Wireless Communications, vol. 6, no. 1, pp. 240-247, 2007.

[18] J. Tubbax, A. Fort, L. V. Der Perre et al., "Joint Compensation of IQ imbalance and Frequency Offset in OFDM systems," in Proceedings of the IEEE Global Telecommunications Conference (GLOBECOM '03), vol. 4, pp. 2365-2369, San Francisco, Calif, USA, December 2003.

[19] G. Xing, M. Shen, and H. Liu, "Frequency offset and I/Q imbalance compensation for direct-conversion receivers," IEEE Transactions on Wireless Communications, vol. 4, no. 2, pp. 673-680, 2005.

[20] C. J. Hsu, R. Cheng, and W. H. Sheen, "Joint least squares estimation of frequency, DC offset, I-Q imbalance, and channel in MIMO receivers," IEEE Transactions on Vehicular Technology, vol. 58, no. 5, pp. 2201-2213, 2009.

[21] Y. J. Chiu and S. P. Hung, "Estimation scheme of the receiver IQ imbalance under carrier frequency offset in com- munication system," IET Communications, vol. 4, no. 11, pp. 1381-1388, 2010.

[22] G.-T. Gil, I.-H. Sohn, J.-K. Park, and Y. H. Lee, "Joint ML estimation of carrier frequency, channel, I/Q mismatch, and DC offset in communication receivers," IEEE Transactions on Vehicular Technology, vol. 54, no. 1, pp. 338-349, 2005.

[23] Y.-H. Chung and S.-M. Phoong, "Joint estimation of transmitter and receiver I/Q imbalances, CFO, and channel response for OFDM systems," in Proceedings of the 1st International Conference on Green Circuits and Systems (ICGCS '10), pp. 247-252, Shanghai, China, June 2010.

[24] D. Tandur and M. Moonen, "Joint adaptive compensation of transmitter and receiver IQ imbalance under carrier frequency offset in OFDM-based systems," IEEE Transactions on Signal Processing, vol. 55, no. 11, pp. 5246-5252, 2007.

[25] J. González-Bayón, C. Carreras, and O. Edfors, "A multistandard frequency offset synchronization scheme for 802.11n, 802.16d, LTE, and DVB-T/H systems," Journal of Computer Systems, Networks, and Communications, vol. 2010, Article ID 628657, 9 pages, 2010.

[26] T. Roman and V. Koivunen, "Carrier frequency synchronization for mobile television receivers," in Proceedings of the IEEE International Symposium on Circuits and Systems (ISCAS '07), pp. 261-264, May 2007.

[27] A. Kiayani, L. Anttila, Y. Zou, and M. Valkama, "Hybird time/ frequency domain compensator for RF impairments in OFDM systems," in Proceedings of the 22nd IEEE International Symposium on Personal, Indoor and Mobile Radio Communications (PIMRC'11), Toronto, Canada, September 2011.

[28] M. Speth, S. Fechtel, G. Fock, and H. Meyr, "Optimum receiver design for OFDM-based broadband transmissionpart II: a case study," IEEE Transactions on Communications, vol. 49, no. 4, pp. 571-578, 2001.

[29] J. Younghwan, K. Jihyun, L. Yuro, A. Jaemin, C. Wongyu, and L. Dongchan, "Additional diversity gain in OFDM receivers under the influence of IQ imbalances," in Proceedings of the IEEE International Conference on Communications (ICC '07), pp. 5915-5920, Glasgow, Scotland, June 2007.

[30] S. Narayanan, B. Narasimhan, and N. Al-Dhahir, "Digital baseband compensation of joint TX/RX I/Q imbalance in mobile MIMO SC-FDE transceivers in the presence of CFO," in Proceedings of the IEEE International Conference on Acoustics, Speech, and Signal Processing (ICASSP '10), pp. 35103513, March 2010.

[31] J. G. Proakis, Digital Communications, McGraw-Hill, New York, NY, USA, 3rd edition, 1995.

[32] L. Deneire, P. Vandenameele, L. Van der Perre, B. Gyselinckx, and M. Engels, "A low-complexity ML channel estimator for OFDM," IEEE Transactions on Communications, vol. 51, no. 2, pp. 135-140, 2003.

[33] S. Haykin, Adaptive Filter Theory, Prentice-Hall, Upper Saddle River, NJ, USA, 4th edition, 2002.

[34] T. B. Sorensen, P. E. Mogensen, and F. Frederksen, "Extension of the ITU channel models for wideband (OFDM) systems," in Proceedings of the IEEE Vehicular Technology Conference (VTC '05), pp. 392-396, Dallas, Tex, USA, September 2005.

[35] 3GPP Technical Specification Group Radio Access Network, "Evolved universal terrestrial radio access (E-UTRA) and evolved universal terrestrial radio access (E-UTRAN); overall description; stage 2," Tech. Rep. TS 36.300, V1.0.0, 2007.

[36] U. Mengali and A. N. D. Andrea, Synchronization Techniques for Digital Receivers, Plenum Press, New York, NY, USA, 1997. 

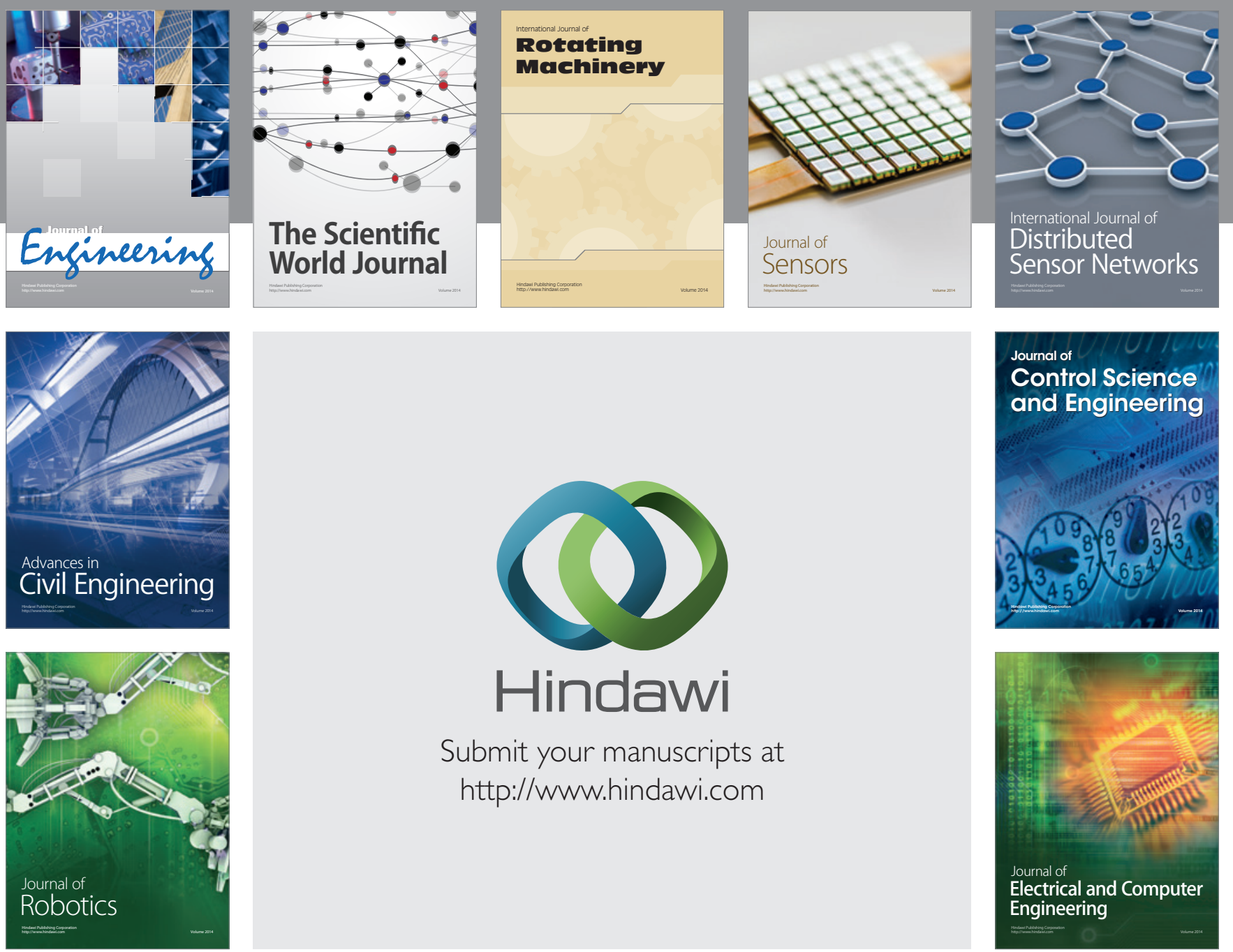

Submit your manuscripts at

http://www.hindawi.com
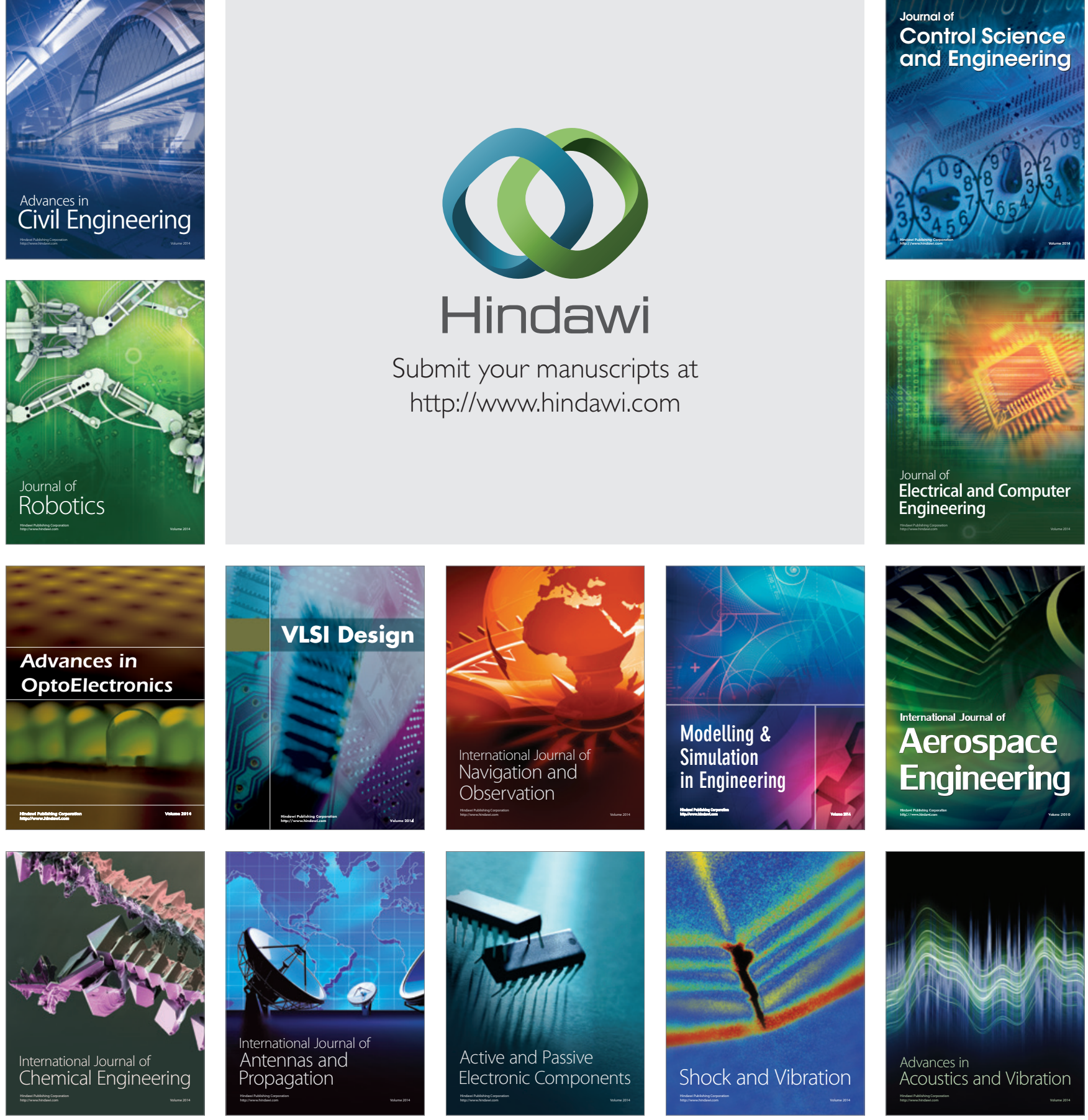